\title{
Derivatives Use
}

\section{Valuation and hedging of contingent claims on power with spikes: A non-Markovian approach}

\author{
Valery A. Kholodnyi \\ Department of Mathematical Sciences, Middle Tennessee State University, \\ Murfreesboro, Tennessee 37132, USA. \\ E-mail: kholodnyi@mtsu.edu
}

Received (in revised form): 16th December, 2004

\begin{abstract}
Valery A. Kholodnyi is Professor of Financial Mathematics and Risk Management as well as Executive Director of the Center for Quantitative Risk Analysis at Middle Tennessee State University. Prior to this, he was Chief Science Officer and Vice President of Research and Development at Integrated Energy Services, Director of Research at TXU Energy Trading and Director of Quantitative Analysis at Reliant Resources. Valery has authored or co-authored three books and over a hundred research papers in finance, mathematics, physics and engineering. He has been an invited speaker at numerous international and national conferences, for both industry practitioners and academic researchers.
\end{abstract}

\section{Practical applications}

As the power markets are becoming deregulated worldwide, the modelling of power prices with spikes is becoming a key problem in energy risk management, physical assets valuation and derivatives pricing. This paper presents and further develops the non-Markovian approach to modelling power prices with spikes proposed in earlier papers by the author. This approach allows for modelling spikes directly as self-reversing jumps which, in turn, allows for the parameters of spikes to be directly observable from market data. This approach also allows for a relatively simple pricing of contingent claims both on power with spikes and on forwards on power for power with spikes. Moreover, contingent claims on forwards on power for power with spikes can be dynamically hedged via the standard delta hedging. For example, European call and put options both on power with spikes and on forwards on power for power with spikes can be priced analytically via simple formulae similar to the Black-Scholes formulae for European call and put options. In turn, this approach also allows for a relatively simple valuation of physical assets such as power plants and transmission lines viewed as real options.

\begin{abstract}
A new approach to modelling spikes in power prices proposed in earlier papers by the author is presented and further developed. By contrast with the standard approaches, power prices with spikes
\end{abstract}

are modelled as a non-Markovian stochastic process that allows for modelling spikes directly as self-reversing jumps. It is also shown how this approach can be used to value and hedge European contingent claims on power with spikes.
Derivatives Use, Trading \& Regulation Vol. 11 No. 4, 2006, pp. 308-333 (c) Palgrave Macmillan Ltd
$1747-4426 / 06 \$ 30.00$ 


\section{INTRODUCTION}

As the power markets are becoming deregulated worldwide, the modelling of spikes in power prices is becoming a key problem in energy risk management, physical assets valuation and derivatives pricing.

This paper presents and further develops a new approach to modelling spikes in power prices proposed by the author in references $1-5$. The main motivation for this approach is that, in the author's opinion, different mechanisms should be responsible for the reversion of power prices to their-long term mean between spikes and for the reversion of power prices to their long-term mean during spikes that is, for the decay of spikes. This is due to the substantial difference in the scales of the deviations of power prices from their long-term mean between spikes and during spikes. For example, power prices in the US Midwest in June 1998 rose to $\$ 7,500$ per megawatt hour (MWh) compared with typical prices of around $\$ 30 / \mathrm{MWh}$ as a result of unseasonably hot weather, planned and unplanned outages and transmission constraints. $^{6}$

By contrast with the standard approaches, ${ }^{6-9}$ this study models power prices with spikes as a non-Markovian stochastic process that allows for modelling spikes directly as self-reversing jumps. In this way, different mechanisms are, in fact, responsible for the reversion of power prices to their long-term mean between spikes and during spikes.

Moreover, it is suggested that employing a Markov process to model power prices with spikes is ultimately making the same mechanism responsible for the reversion of power prices to their-long term mean, both between spikes and during spikes. Indeed, although a Markov process can produce a sharp upward price movement as a suitable jump, it cannot remember the magnitude of this sharp upward price movement separately to produce a shortly followed sharp downward price movement of approximately the same magnitude so that a spike can form.

While the stochastic process proposed is non-Markovian, it can be represented as a product of two Markov processes: the spike process and the process for power prices in the absence of spikes. The spike process is responsible for modelling spikes in power prices. It is either equal to a multiplicative magnitude of a spike during a spike or to unity between spikes. The spike process is constructed with the help of a two-state Markov process in continuous time. This two-state Markov process determines whether power prices are in the spike state, that is, during a spike, or in the inter-spike state, that is, between spikes. If power prices are in the spike state, the multiplicative magnitude of the spike is determined by a random variable with a suitable distribution. It is noted that the spike process constructed in this way, among other things, allows for the parameters of spikes such as duration, frequency and magnitude to be relatively easily estimated directly from market data. The remaining process, the process for power prices in the absence of spikes, is responsible for modelling power prices between spikes.

Although the process proposed is 
non-Markovian, it can be represented as a Markov process with a suitably extended state space that, in addition to the power price, also includes the magnitude of spikes. With the help of this Markov process with the extended state space, a relatively simple analytical expression is obtained for the values of European contingent claims on power with spikes provided that the corresponding European contingent claims can be valued in the case of the process for power prices in the absence of spikes. This, for example, enables a relatively simple analytical expression to be obtained for power forward prices for power with spikes, provided that corresponding power forward prices are known in the case of the process for power prices in the absence of spikes.

Another outcome of the non-Markovian process proposed is that it provides a natural mechanism to explain the absence of spikes in the values of European contingent claims on power far enough from their expiration time while power prices exhibit spikes. As a special case, it is shown that power forward prices far enough from the maturity time of the forward contracts on power do not exhibit spikes, while power prices, - or more precisely power spot prices - do. This enables the value of a European contingent claim on power with spikes to be represented as a correction to the value of the corresponding European contingent claim on power without spikes in a practically important special case of short-lived spikes. A similar correction is also obtained for power forward prices.

Finally, a practically important special case is considered when power forward prices for power without spikes follow a geometric Brownian motion. This is, for example, when power prices in the absence of spikes follow a geometric mean-reverting process. It is shown that, in this case, power forward prices for power with spikes also follow the same geometric Brownian motion far enough from the maturity time of the forward contracts on power. This allows for the valuation of European contingent claims on forwards on power for power with spikes in terms of European contingent claims on forwards on power in the Black-Scholes market environment. This also allows for the dynamic hedging of European contingent claims on forwards on power for power with spikes via the standard delta hedging.

\section{NON-MARKOVIAN PROCESS FOR POWER PRICES WITH SPIKES}

This section defines the non-Markovian process for power prices with spikes as a product of two Markov processes: the spike process and the process for power prices in the absence of spikes. The spike process is responsible for modelling spikes in power prices, and the process for power prices in the absence of spikes is responsible for modelling power prices between spikes.

\section{Two-state Markov process}

As discussed in the Introduction, the spike process is constructed with the help of a two-state Markov process in continuous time. This two-state Markov process will determine whether power prices are in the spike state, that is, during a spike or in the inter-spike state, that is, between spikes. 
Denote this two-state Markov process by $M_{t}, t \geq 0$. Let

$$
P(T, t)=\left(\begin{array}{ll}
P_{s s}(T, t) & P_{s r}(T, t) \\
P_{r s}(T, t) & P_{r r}(T, t)
\end{array}\right), \quad t \leq T
$$

be its $2 \times 2$ transition matrix, where $P_{s s}(T, t)$ and $P_{r s}(T, t)$ are the transition probabilities from the spike state at time $t$ to the spike and inter-spike states at time $T$ and where $P_{s r}(T, t)$ and $P_{r r}(T, t)$ are the transition probabilities from the inter-spike state at time $t$ to the spike and inter-spike states at time $T$. The subscripts $s$ and $r$ stand for the spike state and inter-spike, or regular, state, respectively.

The Kolmogorov-Chapman equation for the Markov process $M_{t}$ can be represented as follows

$$
P(T, t)=P(T, \tau) P(\tau, t), \quad t \leq \tau \leq T
$$

where $P(T, t)$ is the $2 \times 2$ identity matrix whenever $t=T$.

The Markov process $M_{t}$ can also be characterised in terms of its generators, ie $2 \times 2$ matrixes defined as the rate of change of the transition matrix $P(T, t)$ at times $t \geq 0$. It turns out that it is these generators of the Markov process $M_{t}$ that will determine the duration and frequency of spikes, that is, the expected lifetime of spikes and expected time between spikes. In this way, it is these generators of the Markov process $M_{t}$ that can be estimated directly from market data.

More precisely, a one-parameter family $\{L(t): t \geq 0\}$ of $2 \times 2$ real matrices defined by

$$
L(t)=\left.\frac{d}{d T} P(T, t)\right|_{T=t}
$$

is said to generate the Markov process $M_{t}$ and the matrix

$$
L(t)=\left(\begin{array}{ll}
L_{s s}(t) & L_{s r}(t) \\
L_{r s}(t) & L_{r r}(t)
\end{array}\right)
$$

is called a generator.

It can be shown that

$$
\begin{aligned}
& L_{s s}(t)+L_{r s}(t)=0 \text { and } L_{s s}(t) \leq 0, L_{r s}(t) \geq 0 \\
& L_{r r}(t)+L_{s r}(t)=0 \text { and } L_{r r}(t) \leq 0, \quad L_{s r}(t) \geq 0
\end{aligned}
$$

for each time $t \geq 0$. Therefore, each generator $L(t)$ can be characterised by two non-negative real numbers

$$
a(t)=-L_{s s}(t)=L_{r s}(t)
$$

and

$$
b(t)=-L_{r r}(t)=L_{s r}(t)
$$

In terms of the generators of the Markov process $M_{t}$, each transition matrix $P(T, t)$ is given by the following exponential of a matrix

$$
P(T, t)=\mathrm{e}^{\int_{t}^{T} L(\tau) \mathrm{d} \tau}
$$

In the case when the generators $L(t)$ do not commute for different times $t \geq 0$, the exponential of the matrix in (4) has to be replaced by the product integral. (For the definition of a product integral see, for example, Dollard \& Friedmann. ${ }^{10}$ ).

In a practically important special case of a time-homogeneous Markov process $M_{t}$, the transition matrix $P(T, t)$ is, in fact, a function of the difference $T-t$

$$
P(T-t)=\left(\begin{array}{ll}
P_{s s}(T-t) & P_{s r}(T-t) \\
P_{r s}(T-t) & P_{r r}(T-t)
\end{array}\right)
$$


In this case, owing to relationship (2), the generators $L(t)$ are, in fact, time-independent

$$
L=\left(\begin{array}{ll}
L_{s s} & L_{s r} \\
L_{r s} & L_{r r}
\end{array}\right)
$$

and hence relationship (4) takes the following form

$$
P(T-t)=\mathrm{e}^{(T-t) L}, \quad t \leq T
$$

Therefore, in the case of a time-homogeneous Markov process $M_{t}$ with the generator $L s$ the transition matrix $P(T, t)$ is given by

$$
P(T-t)=\left(\begin{array}{c}
\frac{b+a \mathrm{e}^{-(T-t)(a+b)}}{a+b} \frac{b-b \mathrm{e}^{-(T-t)(a+b)}}{a+b} \\
\frac{a-a \mathrm{e}^{-(T-t)(a+b)}}{a+b} \frac{a+b \mathrm{e}^{-(T-t)(a+b)}}{a+b}
\end{array}\right)
$$

where, according to relationship (3), $a \geq 0$ and $b \geq 0$ are given by

$$
a=-L_{s s}=L_{r s} \quad \text { and } \quad b=-L_{r r}=L_{s r}
$$

and where the case of $a+b=0$ is understood as the corresponding limit.

In another practically important special case when the Markov process $M_{t}$ is time-homogeneous on time subintervals, its generators $L(t)$ are time-independent on these time subintervals, hence the transition probabilities $P(T, t)$ can also be found analytically with the help of relationships (1) and (5). In practice, a general two-state Markov process $M_{t}$ can be approximated with any desired degree of accuracy by a two-state Markov process that is time-homogeneous on time subintervals.

Later in the paper, the following decompositions of the transition probabilities of the Markov process $M_{t}$ will be needed

$$
\begin{aligned}
P_{s s}(T, t)= & \mathrm{e}^{\int_{t}^{T} L_{s s}(\tau) d \tau} \\
& +\int_{t}^{T} P_{r s}(\tau, t) L_{s r}(\tau) e^{\int_{t}^{T} L_{s s}(\tau) d \tau^{\prime}} d \tau \\
P_{s r}(T, t)= & \int_{t}^{T} P_{r r}(\tau, t) L_{s r}(\tau) e^{\int_{\tau}^{T} L_{s s}(\tau) d \tau^{\prime}} d \tau
\end{aligned}
$$

To justify the first relationship in (6), note that the event that the Markov process $M_{t}$ is in the spike state at time $T$, given that the process was in the spike state at time $t$, can be decomposed into a disjoint union of the following events:

- The event that the process remains in the spike state for the entire time interval $[t, T]$; and

- The events indexed by $\tau$ in $[t, T]$, where each event with index $\tau$ is the intersection of the following independent events

- The process is in the inter-spike state at time $\tau$ given that the process was in the spike state at time $t$,

- The process transits to the spike state from the inter-spike state during the time interval $[\tau, \tau+\mathrm{d} \tau]$ and

- The process remains in the spike state for the entire time interval $[\tau, T]$.

To justify the second relationship in (6), note that the event that the Markov process $M_{t}$ is in the spike state at time $T$, given that the process was in the inter-spike state at time $t$, can be 
decomposed into a disjoint union of the following events indexed by $\tau$ in $[t, T]$, where each event with index $\tau$ is the intersection of the following independent events:

- The process is in the inter-spike state at time $\tau$ given that the process was in the inter-spike state at time $t$;

- The process transits to the spike state from the inter-spike state during the time interval $[\tau, \tau+\mathrm{d} \tau]$; and

- The process remains in the spike state for the entire time interval $[\tau, T]$.

Note that the first relationship in (6) can also be written as follows

$$
P_{s s}(T, t)=P_{s s}^{s}(T, t)+P_{s s}^{r}(T, t)
$$

where

$$
P_{s s}^{s}(T, t)=\mathrm{e}^{\int_{t}^{T} L_{s s}(\tau) \mathrm{d} \tau}
$$

is the probability that the Markov process $M_{t}$ is in the spike state at time $T$ given that it was in the spike state at time $t$ and remained in the spike state during the entire time interval $[t, T]$, and where

$$
P_{s s}^{r}(T, t)=\int_{t}^{T} P_{r s}(\tau, t) L_{s r}(\tau) \mathrm{e}^{\int_{\tau}^{T} L_{s s}(\tau) \mathrm{d} \tau^{\prime}} \mathrm{d} \tau
$$

is the probability that the Markov process $M_{t}$ is in the spike state at time $T$, given that it was in the spike state at time $t$ and has visited the inter-spike state during the time interval $[t, T]$ at least once.

\section{Spike process}

The spike process that will be responsible for modelling spikes in power prices is now defined.

Let $\xi_{t}>1$ with $t \geq 0$ be independent random variables with the probability density functions $\Xi(t, \xi)$. $\xi_{t}$ will be interpreted as the multiplicative magnitude of spikes that start at time $t$ and $\Xi(t, \xi)$ as the conditional probability density function for the mutiplicative magnitude of spikes that start at time $t$.

The spike process $\lambda_{i} \geq 1$ with $t \geq 0$ is defined as follows. If the Markov process $M_{t}$ is in the inter-spike state, the spike process $\lambda_{t}$ is equal to unity. If the Markov process $M_{t}$ transits into the spike state at time $\tau$, the spike process $\lambda_{t}$ is equal to the value of the random variable $\xi_{\tau}$ during the entire time that the Markov process $M_{t}$ remains in the spike state. Finally, if the Markov process $M_{t}$ is in the spike state at time $t=0$, the spike process $\lambda_{t}$ is initiated at some $\lambda_{0}>1$.

It can be shown ${ }^{1-5}$ that the spike process $\lambda_{t}$ is, in fact, a Markov process and that its transition probability density function $\Lambda\left(t, T, \lambda_{t}, \lambda_{T}\right)$, with the help of relationships in (6), is given in equation 10 where $\delta(x)$ is the Dirac delta function.

$$
\begin{aligned}
& \Lambda\left(t, T, \lambda_{t}, \lambda_{T}\right)= \\
& \left\{\begin{array}{l}
\mathrm{e}_{t}^{\int_{t}^{T} L_{s s}(\tau) d \tau} \delta\left(\lambda_{t}-\lambda_{T}\right)+ \\
\int_{t}^{T} \Xi\left(\tau, \lambda_{T}\right) P_{r s}(\tau, t) L_{s r}(\tau) \mathrm{e}^{\int_{\tau}^{T} L_{s s}(\tau) d \tau^{\prime}} d \tau+\text { if } \lambda_{t}>1 \\
P_{r s}(T, t) \delta\left(1-\lambda_{T}\right) \\
\int_{t}^{T} \Xi\left(\tau, \lambda_{T}\right) P_{r r}(\tau, t) L_{s r}(\tau) \mathrm{e}^{\int_{\tau}^{T} L_{s s}(\tau) d \tau^{\prime}} d \tau+\text { if } \lambda_{t}=1 \\
P_{r r}(T, t) \delta\left(1-\lambda_{T}\right)
\end{array}\right.
\end{aligned}
$$

Finally, the spike process $\lambda_{t}$ is said to be in the spike state or inter-spike state if the Markov process $M_{t}$ is in the spike state or inter-spike state. 


\section{Process for power prices in the absence of spikes}

The process for power prices in the absence of spikes that will be responsible for modelling power prices between spikes is now defined.

Denote by $\hat{\Psi}_{t}>0$ the price (for example, in dollars) of a unit of power (for example, in MWh) at time $t \geq 0$ in the absence of spikes in power prices. Assume that $\hat{\Psi}_{t}$ can be represented as a Markov process. For example, $\hat{\Psi}_{t}$ can be a diffusion process defined by the following stochastic differential equation

$$
\mathrm{d} \hat{\Psi}_{t}=\mu\left(\hat{\Psi}_{t}, t\right) \mathrm{d} t+\sigma\left(\hat{\Psi}_{t}, t\right) \mathrm{d} W_{t},
$$

where $\mu\left(\hat{\Psi}_{t}, \mathrm{t}\right)$ is the drift, $\sigma\left(\hat{\Psi}_{t}, t\right)>0$ is the volatility and $W_{t}$ is the Wiener process. In a practically important special case of the geometric mean-reverting process, the preceding stochastic differential equation takes the following form

$$
\mathrm{d} \hat{\Psi}_{t}=\eta(t)\left[\mu(t)-\ln \hat{\Psi}_{t}\right] \hat{\Psi}_{t} \mathrm{~d} t+\sigma(t) \hat{\Psi}_{t} \mathrm{~d} W_{t}
$$

where $\boldsymbol{\eta}(t)>0$ is the mean-reversion rate, $\boldsymbol{\mu}(t)$ is the equilibrium mean and $\boldsymbol{\sigma}(t)>0$ is the volatility.

\section{Non-Markovian process for power prices with spikes}

Now the non-Markovian process for power prices with spikes can be defined.

Let $\Psi_{t}>0$ be the price (for example, in dollars) of a unit of power (for example, in $\mathrm{MWh}$ ) at time $t \geq 0$ in the presence of spikes in power prices.
Assume that the spike process $\lambda_{t}$ and the process $\hat{\Psi}_{t}$ for power prices in the absence of spikes are independent. Define the process $\Psi_{t}$ with $t \geq 0$ as the product of the spike process $\lambda_{t}$ and the process $\hat{\Psi}_{t}$ for power prices in the absence of spikes

$$
\Psi_{t}=\lambda_{t} \hat{\Psi}_{t}
$$

It is easy to see that $\Psi_{t}$ is a non-Markovian process. Indeed, in order to characterise the future behaviour of the process $\Psi_{t}$, one needs to know the current value of either the process $\lambda_{t}$ or the process $\hat{\Psi}_{t}$ in addition to the current value of the process $\Psi_{t}$. In other words, the state of the power market at any time $t \geq 0$ can be fully characterised by any pair of the values of the processes $\Psi_{t}, \lambda_{t}$, and $\hat{\Psi}_{t}$ at time $t$. Moreover, it can be shown ${ }^{2}$ that, although the process $\Psi_{t}$ is non-Markovian, it can, in fact, be represented as a Markov process that for any time $t \geq 0$ can be fully characterised, for example, by the values of the processes $\lambda_{2}$ and $\hat{\Psi}_{t}$ at time $t$. Equivalently, the non-Markovian process $\Psi_{t}$ can be represented as a Markov process with the extended state space that at any time $t \geq 0$ consists of all possible pairs $\left(\lambda_{t}, \hat{\Psi}_{t}\right)$ with $\lambda_{t} \geq 1$ and $\hat{\Psi}_{t}>0$. This representation of the non-Markovian process $\Psi_{t}$ as a Markov process with the extended state space will be used later in the paper in the valuation of European contingent claims on power with spikes.

The process $\Psi_{t}$ for power prices with spikes is said to be in the spike state or inter-spike state if the spike process $\lambda_{t}$ is in the spike state or inter-spike state or, equivalently, if the Markov process $M_{t}$ is in 
the spike state or inter-spike state.

Now it will be shown that the spike state of the process $\Psi_{t}$ can mimic spikes in power prices ie exhibit sharp upward price movements shortly followed by equally sharp downward price movements of approximately the same magnitude.

Since the expected times $\bar{t}_{s}$ and $\bar{t}_{r}$ for the process $\Psi_{t}$ to be in the spike and inter-spike states starting at time $t$ coincide with those of the Markov process $M_{t}$, one has

$$
\begin{aligned}
& \bar{t}_{s}=\int_{t}^{\infty}(\tau-t) \mathrm{e}^{-\int_{t}^{\tau} a(\tau) \mathrm{d} \tau^{\prime}} a(\tau) d \tau \\
& \bar{t}_{r}=\int_{t}^{\infty}(\tau-t) \mathrm{e}^{-\int_{t}^{\tau} b(\tau) \mathrm{d} \tau^{\prime}} b(\tau) d \tau
\end{aligned}
$$

where $a(t)$ and $b(t)$ are defined in relationship (3).

In a practically important special case of a time-homogeneous Markov process $M_{t}$, relationships in (14) take the following form

$$
\begin{aligned}
& \bar{t}_{s}=\int_{t}^{\infty}(\tau-t) \mathrm{e}^{-a(\tau-t)} a d \tau=\frac{1}{a} \\
& \bar{t}_{r}=\int_{t}^{\infty}(\tau-t) \mathrm{e}^{-b(\tau-t)} b d \tau=\frac{1}{b}
\end{aligned}
$$

so that the expected times $\bar{t}_{s}$ and $\bar{t}_{r}$ for the process $\Psi_{t}$ to be in the spike and inter-spike states starting at time $t$ do not depend on $t$.

If for each time $t \geq 0$ the expected time $\bar{t}_{s}$ for the process $\Psi_{t}$ to be in the spike state is small relative to the characteristic time of change of the process $\hat{\Psi}_{t}$ then the spike state of the process $\Psi_{t}$ can be interpreted as a spike in power prices. For example, if $\hat{\Psi}_{t}$ is a diffusion process defined in (11), the condition mentioned above can be restated as follows

$$
\sigma^{2}\left(\hat{\Psi}_{t}, t\right) \bar{t}_{s}<<1 \text { and } \mu\left(\hat{\Psi}_{t}, t\right) \bar{t}_{s}<<1, t \geq 0
$$

where $\mu\left(\hat{\Psi}_{t}, t\right)$ is the drift and $\sigma\left(\hat{\Psi}_{t}, t\right)$ is the volatility of $\hat{\Psi}_{t}$.

Under this interpretation, $\bar{t}_{s}$ is the expected lifetime of a spike that starts at time $t$, and $\bar{t}_{r}$ is the expected time between two consecutive spikes when the first spike ends at time $t$. In this way, $a(t)$ and $b(t)$ control the duration and frequency of spikes by means of the relationships in (14). For example, it is easy to see with the help of the relationships in (14) that for short-lived spikes $a(t)$ has to be relatively large, while for rare spikes $b(t)$ has to be relatively small.

The spike process $\lambda_{t}$ is now responsible for modelling spikes in power prices. It is either equal to a multiplicative magnitude of a spike during the spike or to unity between spikes. In this way, $\Xi(t, \lambda)$ can be interpreted as the conditional probability density function for the multiplicative magnitude of spikes that start at time $t$. Moreover, the Markov process $M_{t}$ determines whether power prices are in the spike state, either during a spike or in the inter-spike state (between spikes). Finally, since the spike process $\lambda_{t}$ is equal to unity between spikes, the process $\Psi_{t}$ for power prices with spikes — by its definition in relationship (13) coincides between spikes with the process $\hat{\Psi}_{t}$ for power prices in the absences of spikes. Therefore, the process $\hat{\Psi}_{t}$ for power prices in the absence of spikes is responsible for modelling power prices between spikes.

Note that the parameters related to spikes are relatively easy to estimate directly from 
market data. Indeed, under the assumption that the Markov process $M_{t}$ is time-homogeneous, according to the relationships in (15), parameters $a$ and $b$ can be estimated as the inverses of the average lifetime of spikes and the average time between spikes. In practice, as pointed out earlier in this paper, a general two-state Markov process $M_{t}$ can be approximated with any desired degree of accuracy by a two-state Markov process that is time-homogeneous on time subintervals. The estimation of the conditional probability density function $\Xi(t, \lambda)$ for the multiplicative magnitude of spikes can be based on the standard parametric or non-parametric statistical methods. For the parametric statistical methods, scaling or asymptotically scaling probability distributions are of a particular practical importance. ${ }^{1-3}$ Recall that a probability distribution $P(\mathrm{~d} \lambda)$ on the interval $(1, \infty)$ is scaling whenever

$$
P\left(\lambda \geq v \lambda_{0}\right)=v^{-\gamma} P\left(\lambda \geq \lambda_{0}\right), v \geq 1
$$

for some $\gamma>0$. It can be shown that a probability distribution $P(\mathrm{~d} \lambda)$ on the interval $(1, \infty)$ is scaling if, and only if, it is of the form $P\left(\lambda \geq \lambda_{0}\right)=\lambda_{0}{ }^{-\gamma}$ or, equivalently, has the probability density function $p(\lambda)=\gamma \lambda^{-\gamma-1}$. A probability distribution $P(\mathrm{~d} \lambda)$ on the interval $(1, \infty)$ is asymptotically scaling whenever it is scaling asymptotically for large $\lambda_{0}$.

Finally, the process $\Psi_{t}$ for power prices with spikes can also be interpreted in terms of a structural model. ${ }^{1,2,5}$ For example, the Markov process $M_{t}$ can be interpreted as a service-repair process of a representative power plant whose failure at time $t \geq 0$ results in the spike in power prices with the multiplicative magnitude $\lambda_{t}>1$ determined by the conditional probability density function $\Xi(t, \lambda)$. In this way, the spike state and inter-spike state of the Markov process $M_{t}$ can be interpreted as the down state the state when the power plant is not in service while being repaired - and the up state, the state when the power plant is in service. Moreover, $a(t)$ and $b(t)$, which determine the generators of the Markov process $M_{t}$ via the relationships in (3), can be interpreted as the repair rate and failure rate of the power plant at time $t$. In turn, the expected lifetime $\bar{t}_{s}$ of a spike that starts at time $t$ and the expected time $\bar{t}_{r}$ between two consecutive spikes where the first spike ends at time $t$ can be interpreted as the mean down time (mean time to repair) and the mean up time (mean time to failure of the power plant at time $t$ ).

\section{EUROPEAN CONTINGENT CLAIMS ON POWER WITH SPIKES}

This section presents an analytical expression for the values of European contingent claims on power in the case when the dynamics of the power prices with spikes are described by the non-Markovian process $\Psi_{t}$ defined earlier. This assumes that, in the case of the process $\hat{\Psi}_{t}$, the corresponding European contingent claims can be valued for power prices in the absence of spikes. It also shows how the values of European contingent claims on power far enough from their expiration time do not exhibit spikes while the power prices do. 


\section{Valuation of European contingent claims on power without spikes}

In order to value European contingent claims on power in the case of the power prices with spikes following the non-Markovian process $\Psi_{t}$, one needs to value the corresponding European contingent claims in the case of the process $\hat{\Psi}_{t}$ for power prices in the absence of spikes.

For a European contingent claim with inception time $t$, expiration time $T$ and payoff $g$, let $\hat{E}(t, T, g)=\hat{E}(t, T, g)\left(\hat{\Psi}_{t}\right)$ - its value at the inception time $t$ as a function of the power price $\hat{\Psi}_{t}$ at time $t$. By contrast with a European option whose payoff is always non-negative, the payoff of a European contingent claim as a function of the power price can take both positive and negative values.

Assume now that $\hat{\Psi}_{t}$ is a risk-neutral process for the power prices in the absence of spikes. Then the value

$\hat{E}(t, T, g)=\hat{E}(t, T, g)\left(\hat{\Psi}_{t}\right)$ of the European contingent claim with inception time $t$, expiration time $T$ and payoff $g$ can be found as the discounted expected value of its payoff

$$
\begin{aligned}
& \hat{E}(t, T, g)\left(\hat{\Psi}_{t}\right)= \\
& \quad \mathrm{e}^{-\int_{t^{T}(\tau) d \tau}^{\infty} \int_{0}^{\infty} P\left(t, T, \hat{\Psi}_{t}, \hat{\Psi}_{T}\right) g\left(\hat{\Psi}_{T}\right) \mathrm{d} \hat{\Psi}_{T}}
\end{aligned}
$$

where $r(t)$ is the continuously compounded interest rate and $P\left(t, T, \hat{\Psi}_{t}, \hat{\Psi}_{T}\right)$ is the risk-neutral transition probability density function for $\hat{\Psi}_{t}$ as a Markov process.

For example, in the practically important special case of the mean-reverting market environment (re when $\hat{\Psi}_{t}$ follows a geometric mean-reverting process defined in (12)), relationship (16) takes the following form ${ }^{2,11-13}$

$$
\begin{aligned}
& \hat{E}^{M R}(t, T, g)\left(\hat{\Psi}_{t}\right)=\frac{\mathrm{e}^{-\bar{r}(t, T)(T-t)}}{\hat{\sigma}(t, T) \sqrt{2 \pi(T-t)}} \\
& \quad \times \int_{0}^{\infty} \mathrm{e}^{-\frac{1}{2} \frac{\left(a(t, T) \ln \hat{\Psi}_{t}+b(t, T)-\ln \hat{\Psi}_{T}\right)^{2}}{\hat{\sigma}^{2}(t, T)(T-t)}} g\left(\hat{\Psi}_{T}\right) \frac{d \hat{\Psi}_{T}}{\hat{\Psi}_{T}}
\end{aligned}
$$

where

$$
\begin{aligned}
\bar{r}(t, T) & =\frac{1}{T-t} \int_{t}^{T} r(\tau) d \tau \\
\hat{\sigma}(t, T) & =\sqrt{\frac{1}{T-t} \int_{t}^{T} \sigma^{2}(\tau) \mathrm{e}_{t}^{-2 T^{T} \eta(\tau) d \tau^{\prime}} d \tau} \\
a(t, T) & =\mathrm{e}^{-\int_{t}^{T} \eta(\tau) \mathrm{d} \tau} \\
b(t, T) & =\int_{t}^{T} \eta(\tau)\left[\mu(\tau)-\frac{1}{2} \frac{\sigma^{2}(\tau)}{\eta(\tau)}\right] \mathrm{e}^{-\int_{\tau}^{T} \eta(\tau) \mathrm{d} \tau^{\prime}} \mathrm{d} \tau
\end{aligned}
$$

Later in this paper, an expression will be needed for the value of a European contingent claim in the mean-reverting market environment in terms the value of the corresponding European contingent claim in the Black-Scholes market environment, that is, when the risk-neutral dynamics of the price $S_{t}$ of an underlying security are given by the geometric Brownian motion

$$
\mathrm{d} S_{t}=\mu_{B S}(t) S_{t} \mathrm{~d} t+\sigma_{B S}(t) S_{t} \mathrm{~d} W_{t}
$$

where $\mu_{B S}(t)$ is the drift and $\sigma_{B S}(t)>0$ is the volatility.

The value $\hat{E}_{\bar{\sigma}_{B S}, \bar{\mu}_{B S}}^{B S}(t, T, g)=$ $\hat{E}_{\bar{\sigma}_{B S}, \bar{\mu}_{B S}}^{B S}(t, T, g)\left(S_{t}\right)$ in the Black-Scholes market environment of a European contingent claim with inception time $t$, expiration time $T$ and payoff $g$ is given by (see, for example, Wilmott ${ }^{14}$ ) 


$$
\begin{array}{r}
\hat{E}_{\bar{\sigma}_{B S}, \bar{\mu}_{B S}}(t, T, g)\left(S_{t}\right)=\frac{\mathrm{e}^{-\bar{\gamma}(t, T)(T-t)}}{\bar{\sigma}_{B S} \sqrt{2 \pi(T-t)}} \times \\
\int_{0}^{\infty} \mathrm{e}^{-\frac{1}{2} \frac{\left(\ln \left(S_{t} / S_{T}\right)+\left(\bar{\mu}_{B S}-\frac{1}{2} \bar{\sigma}_{B S}^{2}\right)(T-t)\right)^{2}}{\hat{\sigma}_{B S}^{2}(t, T)}} g\left(S_{T}\right) \frac{d S_{T}}{S_{T}}
\end{array}
$$

where

$$
\begin{aligned}
\bar{\sigma}_{B S} & =\sqrt{\frac{1}{T-t} \int_{t}^{T} \sigma_{B S}^{2}(\tau) d \tau} \\
\bar{\mu}_{B S} & =\frac{1}{T-t} \int_{t}^{T} \mu_{B S}(\tau) d \tau
\end{aligned}
$$

It can be shown $n^{2,5,11,12,15}$, with the help of relationships (17) and (18), that the value $\hat{E}^{M R}(t, T, g)$ can be expressed in terms of the value $\hat{E}_{\bar{\sigma}_{B S}, \bar{\mu}_{B S}}^{B S}(t, T, g)$ as follows

$$
\begin{aligned}
\hat{E}^{M R}(t, T, g)\left(\hat{\Psi}_{t}\right)= & \hat{E}_{\hat{\sigma}(t, T), 0}^{B S}(t, T, g) \\
& \left(\hat{\Psi}_{t}^{a(t, T)} \mathrm{e}^{b(t, T)} \mathrm{e}^{(T-t) 1 / 2 \hat{\sigma}^{2}(t, T)}\right)
\end{aligned}
$$

In this way, the values $\hat{C}^{M R}\left(t, T, \hat{\Psi}_{t}, X\right)$ and $\hat{P}^{M R}\left(t, T, \hat{\Psi}_{t}, X\right)$ in the mean-reverting market environment of the European call and put options with inception time $t$, expiration time $T$ and strike $X$ can be expressed in terms of the values $\hat{C}_{\hat{\sigma}(t, T), 0}^{B S}\left(t, T, S_{t}, X\right)$ and $\hat{P}_{\hat{\sigma}(t, T), 0}^{B S}\left(t, T, S_{t}, X\right)$ of the corresponding

European call and put options in the Black-Scholes market environment

$$
\begin{aligned}
& \hat{C}^{M R}\left(t, T, \hat{\Psi}_{t}, X\right)= \\
& \quad \hat{C}_{\hat{\sigma}(t, T), 0}^{B S}\left(t, T, \hat{\Psi}_{t}^{a(t, T)} \mathrm{e}^{b(t, T)} \mathrm{e}^{(T-t) 1 / 2 \hat{\sigma}^{2}(t, T)}, X\right) \\
& \hat{P}^{M R}\left(t, T, \hat{\Psi}_{t}, X\right)= \\
& \quad \hat{P}_{\hat{\sigma}(t, T), 0}^{B S}\left(t, T, \hat{\Psi}_{t}^{a(t, T)} \mathrm{e}^{b(t, T)} \mathrm{e}^{(T-t) 1 / 2 \hat{\sigma}^{2}(t, T)}, X\right)
\end{aligned}
$$

where $\hat{C}_{\bar{\sigma}_{B S}, \bar{\mu}_{B S}}^{B S}\left(t, T, S_{t}, X\right)$ and $\hat{P}_{\bar{\sigma}_{B S}, \bar{\mu}_{B S}}^{B S}$ $\left(t, T, S_{t}, X\right)$ are given by the Black-Scholes formulae

$$
\begin{aligned}
& \hat{C}_{\bar{\sigma}_{B S}, \bar{\mu}_{B S} S}^{B S}\left(t, T, S_{t}, X\right)= \\
& \quad S_{t} \mathrm{e}^{\left(\bar{\mu}_{B S}, \bar{r}(t, T)\right)(T-t)} N\left(d_{+}\right)-X \mathrm{e}^{\bar{r}(t, T)(T-t)} N\left(d_{-}\right) \\
& \hat{P}_{\bar{\sigma}_{B S,}^{B S} \bar{\mu}_{B S}\left(t, T, S_{t}, X\right)=} \\
& \quad X \mathrm{e}^{-\bar{r}(t, T)(T-t)} N\left(-d_{-}\right)-S_{t} \mathrm{e}^{\left(\bar{\mu}_{B S,} \bar{r}(t, T)(T-t)\right.} N\left(-d_{+}\right)
\end{aligned}
$$

with

$$
\begin{aligned}
& d_{ \pm}=\frac{\ln \left(S_{t} / X\right)+\left(\bar{\mu}_{B S} \pm \frac{1}{2} \bar{\sigma}_{B S}^{2}\right)(T-t)}{\bar{\sigma}_{B S} \sqrt{(T-t)}} \\
& N(x)=\frac{1}{\sqrt{2 \pi}} \int_{-\infty}^{x} \mathrm{e}^{-\gamma^{2} / 2} \mathrm{~d} y
\end{aligned}
$$

(See, for example, Wilmott ${ }^{14}$ ).

\section{Valuation of European contingent claims on power with spikes}

An analytical expression is presented for the values of European contingent claims on power in the case when the dynamics of the power prices with spikes are given by the non-Markovian process $\Psi_{t}$.

Consider a European contingent claim on power with inception time $t$, expiration time $T$ and payoff $g$ that explicitly depends, in addition to the power price $\Psi_{I}$ at time $T$, on the state, spike or inter-spike state, of the power price $\Psi_{T}$ and the magnitude of the related spike at time $T$ as determined by the value of the spike process $\lambda_{T}$ at time $T$. The payoff $g$, in view of the definition of process $\Psi_{t}$ in (13), can be represented as a function of any pair of the values of the processes $\Psi_{T}$, $\lambda_{T}$ and $\hat{\Psi}_{T}$ at time $T$. Throughout this paper, payoff $g$ is represented as a function of $\lambda_{T}$ and $\hat{\Psi}_{T}$, that is $g=g_{\lambda_{T}}\left(\hat{\Psi}_{T}\right)=g\left(\lambda_{T} \hat{\Psi}_{T}, \lambda_{T}\right)$. In this way, if the payoff $g$ depends only on the power price $\Psi_{T}=\lambda_{T} \hat{\Psi}_{T}$ at time $T$ and does not explicitly depend on the spike or 
inter-spike state of the power price $\Psi_{T}=\lambda_{T} \hat{\Psi}_{T}$ at time $T$ (as determined by the value of the spike process $\lambda_{T}$ at time $T$ ), then

$$
g_{\lambda_{T}}\left(\hat{\Psi}_{T}\right)=g\left(\lambda_{T} \hat{\Psi}_{T}\right)
$$

A holder of the European contingent claim on power with inception time $t$, expiration time $T$ and payoff $g$ will receive a (positive or negative) payment of either $g_{\lambda_{T}}\left(\hat{\Psi}_{T}\right)=g\left(\lambda_{T} \hat{\Psi}_{T}, \lambda_{T}\right)$ units of account if the power price $\Psi_{T}=\lambda_{T} \hat{\Psi}_{T}$ is in the spike state at time $T$ and the magnitude of the spike is $\lambda_{T}>1$, or $g_{\lambda_{T}=1}\left(\hat{\Psi}_{T}\right)=$ $\left.g\left(\lambda_{T} \hat{\Psi}_{T}, \lambda_{T}\right)\right|_{\lambda_{T}=1}$ units of account if the power price $\Psi_{T}=\lambda_{T} \hat{\Psi}_{T}$ is in the inter-spike state at time $T\left(\lambda_{T}=1\right)$. By allowing the payoffs to depend explicitly on the state, spike or inter-spike state and the magnitude of the related spike, one can construct European contingent claims on power that provide better tailored protection for their holders against spikes in power prices. Moreover, with the help of the beliefs-preferences gauge symmetry introduced by the author, ${ }^{16-22}$ one can construct a dynamically spanning set of European contingent claims with payoffs that allow for the beliefs-preferencesindependent valuation and dynamic replication of European contingent claims on power with spikes. In turn, this allows for the beliefs-preferences- independent valuation and dynamic replication of more general contingent claims on power with spikes, such as universal contingent claims introduced by the author in previous works. ${ }^{2,11,18,23-28}$ These include Bermudan and American options.

As discussed earlier in this paper, the state of the power market at any time $t \geq 0$ can be fully characterised by any pair of the values of the processes $\Psi_{t}, \lambda_{t}$ and $\hat{\Psi}_{t}$ at time $t$. In this regard, the value $E(t, T, g)$ at inception time $t$ of a European contingent claim (on power with spikes) with inception time $t$, expiration time $T$ and payoff $g$ can be represented as a function of any pair of the values of the processes $\Psi_{t}, \lambda_{t}$ and $\hat{\Psi}_{t}$ at time $t$. Throughout this paper, the value $E(t, T, g)$ is represented as a function of $\lambda_{t}$ and $\hat{\Psi}_{t}$, that is $E(t, T, g)=$ $E_{\lambda_{t}}(t, T, g)\left(\hat{\Psi}_{t}\right)=E(t, T, g)\left(\lambda_{t} \hat{\Psi}_{t}, \lambda_{t}\right)$. Note that the value of the European contingent claim (on power with spikes) with inception time $t$, expiration time $T$ and payoff $g$ is either $E_{\lambda_{t}}(t, T, g)\left(\hat{\Psi}_{t}\right)=E(t, T, g)\left(\lambda_{t} \hat{\Psi}_{t}, \lambda_{t}\right)$ units of account if the power price $\Psi_{t}=\lambda_{t} \hat{\Psi}_{t}$ is in the spike state at time $t$ and the magnitude of the spike is $\lambda_{t}>1$ or $E_{\lambda_{t}=1}(t, T, g)\left(\hat{\Psi}_{t}\right)=$ $\left.E(t, T, g)\left(\lambda_{t} \hat{\Psi}_{t}, \lambda_{t}\right)\right|_{\lambda_{t}=1}$ units of account if the power price $\Psi_{t}=\lambda_{t} \hat{\Psi}_{t}$ is in the inter-spike state at time $t$, that is $\lambda_{t}=1$.

As also discussed earlier in this paper, the non-Markovian process $\Psi_{t}$ can be represented as a Markov process with the state space that at any time $t \geq 0$ consists of all possible pairs $\left(\lambda_{t}, \hat{\Psi}_{t}\right)$ with $\lambda_{t} \geq 1$ and $\hat{\Psi}_{t}>0$. Assume now that $\Psi_{t}$ is a risk-neutral process for power prices with spikes so that it can be represented as a risk-neutral Markov process with the state space that at any time $t \geq 0$ consists of all possible pairs $\left(\lambda_{t}, \hat{\Psi}_{t}\right)$ with $\lambda_{t} \geq 1$ and $\hat{\Psi}_{t}>0$. Then the value $E(t, T, g)$ of the European contingent claim (on power with spikes) with inception time $t$, expiration time $T$ and payoff $g$ can be found as the discounted expected value of its payoff. Moreover, it can be shown $n^{1,2,4}$ with the 
help of relationships (10) and (16) that the value $E(t, T, g)$ of the European contingent claim on power with spikes can be represented in terms of the value of the following European contingent claim on power without spikes

$$
\begin{aligned}
& E_{\lambda_{t}}(t, T, g)\left(\hat{\Psi}_{t}\right)=\mathrm{e}^{-\int_{t}^{T} r(\tau) \mathrm{d} \tau} \int_{0}^{\infty} \int_{1}^{\infty}\left[P\left(t, T, \hat{\Psi}_{t}, \hat{\Psi}_{T}\right)\right. \\
& \left.\quad \times \mathrm{L}\left(t, T, \lambda_{t}, \lambda_{T}\right) g_{\lambda_{T}}\left(\hat{\Psi}_{T}\right) \mathrm{d} \lambda_{T} \mathrm{~d} \hat{\Psi}_{T}\right] \\
& \quad=\hat{E}\left(t, T, \bar{g}_{\lambda_{t}}\right)\left(\hat{\Psi}_{t}\right)
\end{aligned}
$$

where the payoff $\bar{g}_{\lambda_{t}}=\bar{g}_{\lambda_{t}}\left(\hat{\Psi}_{T}\right)$ is the risk-neutral average of the payoff $g_{\lambda T}$ over the magnitude of spikes $\lambda_{T}$ at time $T$ given by

$$
\bar{g}_{\lambda_{t}}=\left\{\begin{array}{c}
P_{s s}^{s}(T, t) g_{\lambda_{t}}+\int_{1}^{\infty} \Xi_{s}\left(t, T, \lambda_{T}\right) g_{\lambda_{T}} \mathrm{~d} \lambda_{T} \\
+P_{r s}(T, t) g_{1} \quad \text { if } \lambda_{t}>1 \\
\int_{1}^{\infty} \Xi_{r}\left(t, T, \lambda_{T}\right) g_{\lambda_{T}} \mathrm{~d} \lambda_{T}+P_{r r}(T, t) g_{1} \\
\text { if } \lambda_{t}=1
\end{array}\right.
$$

with

$$
\begin{aligned}
& \Xi_{s}\left(t, T, \lambda_{T}\right)= \\
& \int_{t}^{T} \Xi\left(\tau, \lambda_{T}\right) P_{r s}(\tau, t) L_{s r}(\tau) \mathrm{e}^{\int_{\tau}^{T} L_{s s}\left(\tau^{\prime}\right) \mathrm{d} \tau^{\prime}} \mathrm{d} \tau \\
& \Xi_{r}\left(t, T, \lambda_{T}\right)= \\
& \int_{t}^{T} \Xi\left(\tau, \lambda_{T}\right) P_{r r}(\tau, t) L_{s r}(\tau) \mathrm{e}^{\int_{\tau}^{T} L_{s s}(\tau) \mathrm{d} \tau^{\prime}} \mathrm{d} \tau
\end{aligned}
$$

and where $P_{s s}^{s}(T, t)$ is given in (8).

Owing to the linearity of the value of a European contingent claim as a function of its payoff and relationship (24), the value $E(t, T, g)$ in (23) can also be represented as the risk-neutral average of the value $\hat{E}\left(t, T, g_{\lambda_{T}}\right)$ over the magnitude of spikes $\lambda_{T}$ at time $T$. Since this risk-neutral average can be viewed as a linear combination, possibly continuous, of the values $\hat{E}\left(t, T, g_{\lambda_{T}}\right)$, a European contingent claim on power with spikes can also be viewed as the corresponding portfolio of European contingent claims on power without spikes.

In a special case when the conditional probability density function $\Xi(t, \lambda)$ for the mutiplicative magnitude of spikes that start at time $t$ is independent of time $t$ and is equal to $\Xi(\lambda)$, relationship (24) takes the following form

$$
\bar{g}_{\lambda_{t}}= \begin{cases}P_{s s}^{s}(T, t) g_{\lambda_{t}}+P_{s s}^{r}(T, t) \bar{g}+ & P_{r s}(T, t) g_{1} \\ & \text { if } \lambda_{t}>1 \\ P_{s r}(T, t) \bar{g}+P_{r r}(T, t) g_{1} & \text { if } \lambda_{t}>1\end{cases}
$$

where $\bar{g}$ is the risk-neutral conditional average of the payoff $g_{\lambda_{T}}$ over the magnitude of spikes $\lambda_{T}$ at time $T$ given by

$$
\bar{g}=\int_{1}^{\infty} \Xi\left(\lambda_{T}\right) g_{\lambda_{T}} \mathrm{~d} \lambda_{T}
$$

and where $P_{s s}^{r}(T, t)$ is given in (9).

Moreover, in a special case of spikes with constant magnitude $\lambda$, when the conditional probability density function $\Xi\left(\lambda^{\prime}\right)$ is the Dirac delta function $\delta\left(\lambda-\lambda^{\prime}\right)$, relationship (26) — and hence (24) — take the following form

$$
\bar{g}_{\lambda_{t}}= \begin{cases}P_{s s}(T, t) g_{\lambda}+P_{r s}(T, t) g_{1} & \text { if } \lambda_{t}=\lambda \\ P_{s r}(T, t) g_{\lambda}+P_{r r}(T, t) g_{1} & \text { if } \lambda_{t}=1\end{cases}
$$

where the decomposition in (7) was used.

\section{Linear evolution equation for European contingent claims on power with spikes}

It can be shown ${ }^{2}$ that the value $E(t, T, g)$ of a European contingent claim on power with spikes in (23) satisfies the Cauchy problem for the following linear evolution equation

$$
\begin{aligned}
\frac{\mathrm{d}}{\mathrm{d} t} v+\hat{L}(t) v+\Lambda(t) v-r(t) v & =0, \quad t<T \\
v(T) & =g
\end{aligned}
$$


where $\hat{L}(t)$ and $\Lambda(t)$ are the generators of the process $\hat{\Psi}_{t}$ for power prices in the absence of spikes and of the spike process $\lambda_{t}$ as Markov processes. (For the definition of the generators of a general Markov process see, for example, Dynkin. ${ }^{29}$ )

In a practically important special case when $\hat{\Psi}_{t}$ is the geometric mean-reverting process defined in (12), the generator $\hat{L}(t)$ is given by

$$
\begin{aligned}
\hat{L}(t)= & \frac{1}{2} \sigma^{2}(t) \hat{\Psi}^{2} \frac{\partial^{2}}{\partial \hat{\Psi}^{2}} \\
& +\eta(t)[\mu(t)-\ln \hat{\Psi}] \hat{\Psi} \frac{\partial}{\partial \hat{\Psi}}
\end{aligned}
$$

Also, owing to relationship (10), the generator $\Lambda(t)$ is a linear integral operator with the kernel

$$
\Lambda\left(t, \lambda_{t}, \lambda_{t}^{\prime}\right)=\left\{\begin{array}{r}
L_{s s}(t) \delta\left(\lambda_{t}-\lambda_{t}^{\prime}\right)+L_{r s}(t) \delta\left(1-\lambda_{t}^{\prime}\right) \\
\text { if } \lambda_{t}>1 \\
\Xi\left(t, \lambda_{t}^{\prime}\right) L_{s r}(t)+L_{r r}(t) \delta\left(1-\lambda_{t}^{\prime}\right) \\
\text { if } \lambda_{t}=1
\end{array}\right.
$$

For example, in the special case of spikes with constant magnitude $\lambda$, the generator $\Lambda(t)$ can be represented as the $2 \times 2$ matrix $L^{*}(t)$ transposed to the generator $L(t)$ in (2) of the Markov process $M_{t}$. In turn, $v=v(t)$ and $g$ can be represented as two-dimensional vector functions

$$
v(t)=\left(\begin{array}{l}
E_{\lambda_{t}=\lambda}(t, T, g) \\
E_{\lambda_{t}=1}(t, T, g)
\end{array}\right) \text { and } g=\left(\begin{array}{l}
g_{\lambda_{T}=\lambda} \\
g_{\lambda_{T}=1}
\end{array}\right)
$$

It should be pointed out that $\Lambda(t)$ represented as $L^{*}(t)$ can also be expressed in terms of the Pauli matrices, which gives rise to an analogy between the preceding linear evolution equation for European contingent claims on power with spikes and the Schrödinger equation for a two-component spinor describing a non-relativistic spin $1 / 2$ particle in an electromagnetic field. (For the definition of the Pauli matrices, the Schrödinger equation and related terminology see, for example, Cohen-Tannoudji et al. ${ }^{30}$.

\section{Why values of European contingent claims on power do not exhibit spikes while power prices do}

It will be shown ${ }^{1,2,4,5}$ that when the time to expiration of a European contingent claim on power is relatively large with respect to the duration of spikes, the value of this European contingent claim does not exhibit spikes, while the power prices do.

For simplicity, a special case is considered when the spikes have constant magnitude $\lambda$ and the Markov process $M_{t}$ is time-homogeneous. In this case, the value $E(t, T, g)$ of the European contingent claim with inception time $t$, expiration time $T$ and payoff $g$ is determined by relationship (23) with the payoff $\bar{g}_{\lambda_{t}}$ given by (28) and the transition matrix $P(T, t)=P(T-t)$ of the Markov process $M_{t}$ is given by (5).

With the help of relationship (5), the transition probabilities of the Markov process $M_{t}$ can be represented for large $T-t$ as follows

$$
\begin{aligned}
& P_{s s}(T-t)=\pi_{s}+O\left(\mathrm{e}^{-(T-t) a}\right), \\
& P_{s r}(T-t)=\pi_{s}+O\left(\mathrm{e}^{-(T-t) a}\right) \\
& P_{r s}(T-t)=\pi_{r}+O\left(\mathrm{e}^{-(T-t) a}\right), \\
& P_{r r}(T-t)=\pi_{r}+O\left(\mathrm{e}^{-(T-t) a}\right)
\end{aligned}
$$


where

$$
\pi_{s}=\frac{b}{a+b} \text { and } \pi_{r}=\frac{a}{a+b}
$$

are the ergodic probabilities of the Markov process $M_{t}$ to be in the spike and inter-spike states and where $O\left(\mathrm{e}^{-(T-t) a}\right)$ stands for a term of the order $\mathrm{e}^{-(T-t) a}$ or higher.

Therefore, in view of relationship (29) the payoffs $\bar{g}_{\lambda_{t}=\lambda}$ and $\bar{g}_{\lambda_{t}=1}$ in (28) coincide up to the terms of the order $O\left(\mathrm{e}^{-(T-t) a}\right)$

$$
\bar{g}_{\lambda_{t}=\lambda}=\bar{g}_{\text {erg }}+O\left(\mathrm{e}^{-(T-t) a}\right)
$$

and

$$
\bar{g}_{\lambda_{t}=1}=\bar{g}_{\text {erg }}+O\left(\mathrm{e}^{-(T-t) a}\right)
$$

where $\bar{g}_{\text {erg }}$ is the risk-neutral ergodic average of the payoff $g_{\lambda_{T}}$ over the magnitude of spikes $\lambda_{T}$ at time $T$ given by

$$
\bar{g}_{\text {erg }}=\pi_{s} g_{\lambda_{T}=\lambda}+\pi_{r} g_{\lambda_{T}=1}
$$

with the ergodic probabilities $\pi_{s}$ and $\pi_{r}$ given in (30).

In turn, the values $E_{\lambda_{t}=\lambda}(t, T, g)$ and $E_{\lambda_{\mathrm{t}}=1}(t, T, g)$ determined by relationship (23) with the payoffs $\bar{g}_{\lambda_{t}=\lambda}$ and $\bar{g}_{\lambda_{t}=1}$ given by (31) also coincide up to the terms of the order $O\left(\mathrm{e}^{-(T-t) a}\right)$ and hence can be combined into a single expression as follows

$$
\begin{aligned}
E(t, T, g)\left(\hat{\Psi}_{t}\right)= & \hat{E}\left(t, T, \bar{g}_{\text {erg }}\right)\left(\hat{\Psi}_{t}\right) \\
& +O\left(\mathrm{e}^{-(T-t) a}\right),
\end{aligned}
$$

where $E(t, T, g)$ is either $E_{\lambda_{t}=\lambda}(t, T, g)$ or $E_{\lambda_{t}=1}(t, T, g)$.

In this regard, if the time to expiration
$T-t$ of a European contingent claim on power with spikes is relatively large with respect to the expected lifetime of a spike $\bar{t}_{s}=1 / a$, the values $E_{\lambda_{t}=\lambda}(t, T, g)$ and $E_{\lambda_{t=1}}(t, T, g)$ of the European contingent claim during a spike and between spikes differ only by an exponentially small term of the order $O\left(\mathrm{e}^{-(T-t) a}\right)$. As a result, the value of a European contingent claim on power does not exhibit spikes, while power prices do. In turn, the value of a European contingent claim can start exhibiting spikes when its time to expiration $T-t$ approaches the expected lifetime of a spike $\bar{t}_{s}=1 / a$.

In view of relationship (32), the value $E(t, T, g)$ in (33) can also be represented in terms of the risk-neutral ergodic average of the values $\hat{E}\left(t, T, g_{\lambda_{\tau}}\right)$ over the magnitude of spikes $\lambda_{T}$ at time $T$

$$
\begin{aligned}
E(t, T, g)\left(\hat{\Psi}_{t}\right)= & \pi_{s} \hat{E}\left(t, T, g_{\lambda_{T}=\lambda}\right)\left(\hat{\Psi}_{t}\right) \\
& +\pi_{r} \hat{E}\left(t, T, g_{\lambda_{T}=1}\right)\left(\hat{\Psi}_{t}\right) \\
& +O\left(\mathrm{e}^{-(T-t) a}\right)
\end{aligned}
$$

For example, it can be shown ${ }^{2}$ that, in the case of the European call and put options with strike $X$, relationship (34) take the following form

$$
\begin{aligned}
C\left(t, T, \hat{\Psi}_{t}, X\right)= & \pi_{s} \lambda \hat{C}\left(t, T, \hat{\Psi}_{t}, \lambda^{-1} X\right) \\
& +\pi_{r} \hat{C}\left(t, T, \hat{\Psi}_{t}, X\right) \\
& +O\left(\mathrm{e}^{-(T-t) a}\right) \\
P\left(t, T, \hat{\Psi}_{t}, X\right)= & \pi_{s} \lambda \hat{P}\left(t, T, \hat{\Psi}_{t}, \lambda^{-1} X\right) \\
& +\pi_{r} \hat{P}\left(t, T, \hat{\Psi}_{t}, X\right) \\
& +O\left(\mathrm{e}^{-(T-t) a}\right)
\end{aligned}
$$

In view of the interpretation of the process $\Psi_{t}$ for power prices with spikes in terms of the structural model discussed earlier in this paper, the ergodic probabilities $\pi_{s}$ and $\pi_{r}$ 
can be interpreted as the unavailability and availability of a representative power plant.

The unavailability, or the forced outage rate, of a power plant is the ratio of the forced outage hours to the total of the in-service hours and the forced outage hours. Similarly, the availability of a power plant is the ratio of the in-service hours to the total of the in-service hours and the forced outage hours. In this way, the value $E(t, T, g)$ in (34) of a European contingent claim on power with spikes can be represented in terms of the weighted sum of the values $\hat{E}\left(t, T, g_{\lambda_{T}=\lambda}\right)$ and $\hat{E}\left(t, T, g_{\lambda_{T}=1}\right)$ of European contingent claims that expire during a spike and between spikes with weights equal to the unavailability and availability of a representative power plant.

Furthermore, consider a practically important special case of short-lived spikes, that is, when the expected lifetime of a spike $\bar{t}_{s}=1 / a$ is relatively short with respect to the expected time between spikes $\bar{t}_{r}=1 / b$. Then the ergodic probabilities $\pi_{s}$ and $\pi_{r}$ given by relationship (30) can be represented as follows

$$
\pi_{s}=t_{c h}+o\left(t_{c h}\right)
$$

and

$$
\pi_{r}=1-t_{c h}+o\left(t_{c h}\right)
$$

where $t_{c h}=b / a=\bar{t}_{s} / \bar{t}_{r}$ is the characteristic lifetime of a spike introduced by the author, ${ }^{1-5}$ and where $o\left(t_{c h}\right)$ stands for a term of the order higher than $t_{c h}$.

With the help of relationship (36) the value $E(t, T, g)$ in (33) of the European contingent claim on power with spikes can be represented ${ }^{1,2}$ as the following correction to the value $\hat{E}\left(t, T, g_{\lambda_{T}=1}\right)$ of the corresponding European contingent claim on power without spikes

$$
\begin{aligned}
& E(t, T, g)\left(\hat{\Psi}_{t}\right)=\hat{E}\left(t, T, g_{\lambda_{T}=1}\right)\left(\hat{\Psi}_{t}\right) \\
& \quad+t_{c h} \hat{E}\left(t, T, g_{\lambda_{T}=\lambda}-g_{\lambda_{T}=1}\right)\left(\hat{\Psi}_{t}\right)+o\left(t_{c h}\right) .
\end{aligned}
$$

For example, in the case of the European call and put options with strike $X$, the preceding relationship (37) takes the following form ${ }^{1,2}$

$$
\begin{aligned}
C\left(t, T, \hat{\Psi}_{t}, X\right)= & \hat{C}\left(t, T, \hat{\Psi}_{t}, X\right) \\
& +t_{c h}\left[\lambda \hat{C}\left(t, T, \hat{\Psi}_{t}, \lambda^{-1} X\right)\right. \\
& \left.-\hat{C}\left(t, T, \hat{\Psi}_{t}, X\right)\right]+o\left(t_{c h}\right) \\
P\left(t, T, \hat{\Psi}_{t}, X\right)= & \hat{P}\left(t, T, \hat{\Psi}_{t}, X\right) \\
& +t_{c h}\left[\lambda \hat{P}\left(t, T, \hat{\Psi}_{t}, \lambda^{-1} X\right)\right. \\
& \left.-\hat{P}\left(t, T, \hat{\Psi}_{t}, X\right)\right]+o\left(t_{c h}\right)
\end{aligned}
$$

The presented analysis in the special case of spikes with constant magnitude $\lambda$ and the time-homogeneous Markov process $M_{t}$ can be extended to the general case. ${ }^{1,2,4}$ For example, in the case of the time-independent conditional probability density function $\Xi(\lambda)$ relationships (31)-(34) and (37) are still valid if $g_{\lambda_{T}=\lambda}$ is replaced by the conditional average $\bar{g}$ of the payoff $g_{\lambda_{T}}$ over the magnitude of spikes $\lambda_{T}$ at time $T$ given in (27).

Finally, consider the following practically important special case when the risk-neutral dynamics of power prices $\hat{\Psi}_{t}$ in the absence of spikes are given by a geometric mean-reverting process defined in (12). In this case, relationship (33) takes the following form

$$
\begin{aligned}
E(t, T, g)\left(\hat{\Psi}_{t}\right)= & \hat{E}^{M R}\left(t, T, \bar{g}_{\text {erg }}\right)\left(\hat{\Psi}_{t}\right) \\
& +O\left(\mathrm{e}^{-(T-t) a}\right)
\end{aligned}
$$


where the value of the European contingent claim in the mean-reverting market environment can be found with the help of relationship (17). For example, if the payoff $g$ is of the form (22) such that

$$
g\left(\lambda_{T} \hat{\Psi}_{T}\right)=\sum_{n=1}^{N} g_{n} \lambda_{T}^{\omega_{n}} \hat{\Psi}_{T}^{\omega_{n}}
$$

where $g_{n}$ and $\omega_{n}$ are real numbers, then it can be shown ${ }^{1,2}$ with the help of the method of eigenclaims that relationship (39) takes the following form

$$
\begin{aligned}
& E(t, T, g)\left(\hat{\Psi}_{t}\right)=\sum_{n=1}^{N}\left[\left(\pi_{s} \lambda^{\omega_{n}}+\pi_{r}\right)\right. \\
& \left.\quad \times g_{n} \mathrm{e}^{(T-t)\left(1 / 2 \hat{\sigma}^{2}(t, T) \omega_{n}^{2}-\bar{r}(t, T)\right)} \mathrm{e}^{\omega_{n} b(t, T)} \hat{\Psi}_{t}^{\omega_{n} a(t, T)}\right] \\
& \quad+O\left(\mathrm{e}^{-(T-t) a}\right)
\end{aligned}
$$

The method and the notion of eigenclaims were introduced by the author ${ }^{11}$ and, to the best of our knowledge, for the first time applied to the valuation of power-sensitive contingent claims in Kholodnyi. ${ }^{1-3,12,13,15,18,31-34}$ If the payoff of a general European contingent claim on power with spikes can be approximated, in a suitable sense, by payoffs $g$ in (40), its value in (39) can also be approximated, in a suitable sense, by $E(t, T, g)$ in the preceding relationship. Since $E(t, T, g)$ in the preceding relationship is determined analytically, the value of this general European contingent claim can also be approximated analytically. It is also pointed out $^{1,2}$ that, in the case when the time-independent conditional probability density function $\Xi(\lambda)$ corresponds to a scaling probability distribution, that is, $\Xi(\lambda)$ is of the form $\gamma \lambda^{-\gamma-1}$ for some $\gamma>0$, the proceeding relationship can be modified as follows

$$
\begin{aligned}
& E(t, T, g)\left(\hat{\Psi}_{t}\right)=\sum_{n=1}^{N}\left[\left(\pi_{s} \frac{\gamma}{\gamma-\omega_{n}}+\pi_{r}\right)\right. \\
& \left.\quad \times g_{n} \mathrm{e}^{(T-t)\left[1 / 2 \hat{\sigma}^{2}(t, T) \omega_{n}^{2}-\bar{r}(t, T)\right]} \mathrm{e}^{\omega_{n} b(t, T)} \hat{\Psi}_{t}^{\omega_{n}(t, T)}\right] \\
& \quad+O\left(\mathrm{e}^{-(T-t) a}\right)
\end{aligned}
$$

where $\gamma>\omega_{n}$.

Moreover, in the case under consideration, relationship (35) takes the following form

$$
\begin{aligned}
C\left(t, T, \hat{\Psi}_{t}, X\right)= & \pi_{s} \lambda \hat{C}^{M R}\left(t, T, \hat{\Psi}_{t}, \lambda^{-1} X\right) \\
& +\pi_{r} \hat{C}^{M R}\left(t, T, \hat{\Psi}_{t}, X\right) \\
& +O\left(\mathrm{e}^{-(T-t) a}\right) \\
P\left(t, T, \hat{\Psi}_{t}, X\right)= & \pi_{s} \lambda \hat{P}^{M R}\left(t, T, \hat{\Psi}_{t}, \lambda^{-1} X\right) \\
& +\pi_{r} \hat{P}^{M R}\left(t, T, \hat{\Psi}_{t}, X\right) \\
& +O\left(\mathrm{e}^{-(T-t) a}\right)
\end{aligned}
$$

where the values of the European call and put options in the mean-reverting market environment can be found with the help of relationships (20) and (21). In view of relationships (19) and (18), the value in (39) of a European contingent claim on power with spikes can be represented in terms of the value of a European contingent claim in the Black-Scholes market environment. For example, in view of relationship (20), the values in (41) of European call and put options on power with spikes can be represented in terms of the values of European call and put options in the Black-Scholes market environment that are given by the Black-Scholes formulas in (21). Note also that relationships (37) and (38) in the case under consideration can be analysed similarly. 


\section{MODELLING POWER FORWARD PRICES FOR POWER WITH SPIKES}

This section presents an analytical expression for the power forward prices in the case when the dynamics of the power prices, or more precisely power spot prices, with spikes are described by the non-Markovian process $\Psi_{t}$ defined earlier in the paper. This is assuming that the corresponding power forward prices are known in the case of the process $\hat{\Psi}_{t}$ for power prices in the absence of spikes. It is also shown how the power forward prices far enough from the maturity time of the forward contracts on power do not exhibit spikes, while the power prices do. This allows for the analytical valuation and dynamic hedging of European contingent claims on forwards on power in a practically important special case when $\hat{\Psi}_{t}$ is a geometric mean-reverting process.

\section{Power forward prices for power without spikes}

In order to find power forward prices in the case of the power prices, or, more precisely, power spot prices, with spikes following the non-Markovian process $\Psi_{t}$, one needs to find the corresponding power forward prices in the case of the process $\hat{\Psi}_{t}$ for power prices in the absence of spikes.

Assume now that $\hat{\Psi}_{t}$ is a risk-neutral process for power prices in the absence of spikes. Then the power forward price $\hat{F}(t, T)=\hat{F}(t, T)\left(\hat{\Psi}_{t}\right)$ at time $t$ for the forward contract with maturity time $T$ can be found as the risk-neutral expected value of the power prices $\hat{\Psi}_{T}$ at time $T$

$$
\hat{F}(t, T)\left(\hat{\Psi}_{t}\right)=\int_{0}^{\infty} P\left(t, T, \hat{\Psi}_{t}, \hat{\Psi}_{T},\right) \hat{\Psi}_{T} \mathrm{~d} \hat{\Psi}_{T}
$$

where $P\left(t, T, \hat{\Psi}_{t}, \hat{\Psi}_{T}\right)$ is the risk-neutral transition probability density function for $\hat{\Psi}_{t}$ as a Markov process.

For example, in a practically important special case when the risk-neutral dynamics of power prices $\hat{\Psi}_{t}$ in the absence of spikes are given by a geometric mean-reverting process defined in (12), relationship (42) takes the following form ${ }^{3,5,11,15}$

$$
\hat{F}(t, T)\left(\hat{\Psi}_{t}\right)=\mathrm{e}^{(T-t) 1 / 2 \hat{\sigma}^{2}(t, T)} \mathrm{e}^{b(t, T)} \hat{\Psi}_{t}^{a(t, T)}
$$

where $\hat{\sigma}(t, T), a(t, T)$ and $b(t, T)$ are given in (17). Moreover, the risk-neutral dynamics of the power forward prices $\hat{F}(t, T)$ are given by the geometric Brownian motion with the zero drift

$$
\mathrm{d} \hat{F}(t, T)=\sigma_{\hat{F}}(t) \hat{F}(t, T) \mathrm{d} W_{t}
$$

where the volatility $\sigma_{\hat{F}}(t)>0$ is given by

$$
\sigma_{\hat{F}}(t)=\sigma(t) \mathrm{e}^{-\int_{t}^{T} \eta(\tau) \mathrm{d} \tau}
$$

\section{Power forward prices for power with spikes}

An analytical expression is now presented for the power forward prices in the case when the dynamics of the power prices, or more precisely power spot prices, with spikes are described by the non-Markovian process $\Psi_{t}$.

As discussed earlier in this paper, the state of the power market at any time $t \geq 0$ can be fully characterised by any pair of the values of the processes $\Psi_{t}, \lambda_{t}$ and $\hat{\Psi}_{t}$ at time $t$. In this regard, the power forward price $F(t, T)$ at time $t$ for the forward contract (on power with spikes) with maturity time $T$ can be represented as a 
function of any pair of the values of the processes $\Psi_{t}, \lambda_{t}$ and $\hat{\Psi}_{t}$ at time $t$.

Throughout this paper, $F(t, T)$ is represented as a function of $\lambda_{t}$ and $\hat{\Psi}_{t}$, that is

$F(t, T)=F_{\lambda_{t}}(t, T)\left(\hat{\Psi}_{t}\right)=F(t, T)\left(\lambda_{t} \hat{\Psi}_{t}, \lambda_{t}\right)$. Note that the power forward price $F(t, T)$ at time $t$ for the forward contract (on power with spikes) with maturity time $T$ is either $F_{\lambda_{t}}(t, T),\left(\hat{\Psi}_{t}\right)=F(t, T)\left(\lambda_{t} \hat{\Psi}_{t}, \lambda_{t}\right)$ units of account if the power price $\Psi_{t}=\lambda_{t} \hat{\Psi}_{t}$ is in the spike state at time $t$ and the magnitude of the spike is $\lambda_{t}>1$, or $F_{\lambda_{t}=1}(t, T)\left(\hat{\Psi}_{t}\right)=\left.F(t, T)\left(\lambda_{t} \hat{\Psi}_{t}, \lambda_{t}\right)\right|_{\lambda_{t}=1}$ units of account if the power price $\Psi_{t}=\lambda_{t} \hat{\Psi}_{t}$ is in the inter-spike state at time $t$, (re $\left.\lambda_{t}=1\right)$.

As also discussed earlier in the paper, the non-Markovian process $\Psi_{t}$ can be represented as a Markov process with the state space that at any time $t \geq 0$ consists of all possible pairs $\left(\lambda_{t}, \hat{\Psi}_{t}\right)$ with $\lambda_{t} \geq 1$ and $\hat{\Psi}_{t}>0$. Assume now that $\Psi_{t}$ is a risk-neutral process for power prices with spikes so that it can be represented as a risk-neutral Markov process with the state space that at any time $t \geq 0$ consists of all possible pairs $\left(\lambda_{t}, \hat{\Psi}_{t}\right)$ with $\lambda_{t} \geq 1$ and $\hat{\Psi}_{t} .>0$. Then the power forward price $F(t, T)$ at time $t$ for the forward contract (on power with spikes) with maturity time $T$ can be found as the risk-neutral expected value of the power prices $\Psi_{t}$ at time T. Moreover, it can be shown, ${ }^{2,3}$ with the help of relationships (10) and (42), that the power forward price $F(t, T)$ for power with spikes can be expressed in terms of the corresponding power forward price $\hat{F}(t, T)$ for power without spikes

$$
F_{\lambda_{t}}(t, T)\left(\hat{\Psi}_{t}\right)=\bar{\lambda}_{\lambda_{t}}(t, T) \hat{F}(t, T)\left(\hat{\Psi}_{t}\right)
$$

where $\bar{\lambda}_{\lambda_{t}}$ is the risk-neutral average magnitude of spikes at time $T$ given by

$$
\bar{\lambda}_{\lambda_{t}}(t, T)=\left\{\begin{array}{rr}
P_{s s}^{s}(T, t) \lambda_{t}+\int_{1}^{\infty} \Xi_{s}\left(t, T, \lambda_{T}\right) \lambda_{T} \mathrm{~d} \lambda_{T} \\
+P_{r s}(T, t) & \text { if } \lambda_{t}>1 \\
\int_{1}^{\infty} \Xi_{r}\left(t, T, \lambda_{T}\right) \lambda_{T} d \lambda_{T}+P_{r r}(T, t) & \text { if } \lambda_{t}=1 \\
& (46)
\end{array}\right.
$$

where $P_{s s}^{s}(T, t)$ is given in (8) and $\Xi_{s}\left(t, T, \lambda_{T}\right)$ and $\Xi_{r}\left(t, T, \lambda_{T}\right)$ are given in (25).

In a special case when the conditional probability density function $\Xi(t, \lambda)$ for the mutiplicative magnitude of spikes that start at time $t$ is independent of time $t$ and is equal to $\Xi(\lambda)$, relationship (46) takes the following form

$$
\bar{\lambda}_{\lambda_{t}}(t, T)=\left\{\begin{array}{lr}
P_{s s}^{s}(T, t) \lambda_{t}+P_{s s}^{r}(T, t) \bar{\lambda}+P_{r s}(T, t) \\
P_{s r}(T, t) \bar{\lambda}+P_{r r}(T, t) & \text { if } \lambda_{t}>1 \\
& \text { if } \lambda_{t}=1
\end{array}\right.
$$

where $\bar{\lambda}$ is the risk-neutral conditional average magnitude of spikes given by

$$
\bar{\lambda}=\int_{1}^{\infty} \Xi(\lambda) \lambda \mathrm{d} \lambda
$$

and where $P_{s s}^{r}(T, t)$ is given in (9).

For example, ${ }^{2,3}$ in a practically important special case when the conditional probability density function $\Xi(\lambda)$ corresponds to a scaling probability distribution - that is, where $\Xi(\lambda)$ is of the form $\gamma \lambda^{-\gamma-1}$ for some $\gamma>0$ - the conditional average magnitude of spikes $\bar{\lambda}$ in (48) is given by

$$
\bar{\lambda}=\frac{\gamma}{\gamma-1}
$$

where $\lambda>1$. 
Moreover, in a special case of spikes with constant magnitude $\lambda$ - when the conditional probability density function $\Xi\left(\lambda^{\prime}\right)$ is the Dirac delta function $\delta\left(\lambda-\lambda^{\prime}\right)$ - relationship (47), and hence (46), take the following form

$$
\bar{\lambda}_{\lambda_{t}}(t, T)= \begin{cases}P_{s s}(T, t) \lambda+P_{r s}(T, t) & \text { if } \lambda_{t}=1 \\ P_{s r}(T, t) \lambda+P_{r r}(T, t) & \text { if } \lambda_{t}=1\end{cases}
$$

Finally, note that it is with the help of the expression in (45) for the power forward prices $F(t, T)$ for power with spikes that the risk-neutral process $\Psi_{t}$ for power prices with spikes can be calibrated to market data.

\section{Why power forward prices do not exhibit spikes, while power prices do}

It will be shown ${ }^{2,3,5}$ that, when the time to maturity of a forward contract on power is relatively large with respect to the duration of spikes, the power forward prices do not exhibit spikes while the power prices, or more precisely power spot prices, do.

For simplicity, again a special case is considered when the spikes have constant magnitude $\lambda$, and the Markov process $M_{t}$ is time-homogeneous. In this case, the power forward price $F(t, T)$ at time $t$ for the forward contract with maturity time $T$ is determined by relationship (45), with the average magnitude of spikes $\bar{\lambda}_{\lambda_{t}}(t, T)$ given by (49), and the transition matrix $P(T, t)=P(T-t)$ of the Markov process $M_{t}$ given by (5).

In view of relationship (29), the average magnitudes of spikes $\bar{\lambda}_{\lambda_{t}=\lambda}(t, T$,$) and$ $\bar{\lambda}_{\lambda_{t}=1}(t, T$,$) given by (49) coincide up to the$ terms of the order $O\left(\mathrm{e}^{-(T-t) a}\right)$

$$
\bar{\lambda}_{\lambda_{t}=\lambda}(t, T)=\bar{\lambda}_{\mathrm{erg}}+O\left(\mathrm{e}^{-(T-t) a}\right)
$$

and

$$
\bar{\lambda}_{\lambda_{t}=1}(t, T)=\bar{\lambda}_{\text {erg }}+O\left(\mathrm{e}^{-(T-t) a}\right)
$$

where $\bar{\lambda}_{\text {erg }}$ is the risk-neutral ergodic average magnitude of spikes given by

$$
\bar{\lambda}_{\mathrm{erg}}=\pi_{s} \lambda+\pi_{r}
$$

with the ergodic probabilities $\pi_{\mathrm{s}}$ and $\pi_{r}$ given in (30).

In turn, the power forward prices $F_{\lambda_{t}=\lambda}(t, T)\left(\hat{\Psi}_{t}\right)$ and $F_{\lambda_{t}=1}(t, T)\left(\hat{\Psi}_{t}\right)$ determined by relationship (45) with the average magnitude of spikes $\bar{\lambda}_{\lambda_{t}=\lambda}(t, T)$ and $\bar{\lambda}_{\lambda_{t}=1}(t, T)$ given by (50) also coincide up to the terms of the order $O\left(\mathrm{e}^{-(T-t) a}\right)$ and hence can be combined into a single expression as follows

$$
\begin{aligned}
F(t, T)\left(\hat{\Psi}_{t}\right)= & \bar{\lambda}_{\operatorname{erg}} \hat{F}(t, T)\left(\hat{\Psi}_{t}\right) \\
& +O\left(\mathrm{e}^{-(T-t) a}\right)
\end{aligned}
$$

where $F(t, T)\left(\hat{\Psi}_{t}\right)$ is either $F_{\lambda_{t}=\lambda}(t, T)\left(\hat{\Psi}_{t}\right)$ or $F_{\lambda_{t}=1}(t, T)\left(\hat{\Psi}_{t}\right)$.

In this regard, if the time to maturity $T-t$ of the forward contract on power is relatively large with respect to the expected lifetime of a spike $\bar{t}_{s}=1 / a$, the power forward prices $F_{\lambda_{t}=\lambda}(t, T)\left(\hat{\Psi}_{t}\right)$ and $F_{\lambda_{t}=1}(t, T)\left(\hat{\Psi}_{t}\right)$ during a spike and between spikes differ only by an exponentially small term of the order $O\left(\mathrm{e}^{-(T-t) a}\right)$. As a result, the power forward prices do not exhibit spikes, while the power prices do. In turn, the power forward prices can start exhibiting spikes and resembling the power prices when the forward contract nears its maturity with the time to maturity $T-t$ 
approaching the expected lifetime of a spike $\bar{t}_{s}=1 / a$.

For example, consider a practically important special case when, in the absence of spikes in power prices, the risk-neutral dynamics of power forward prices $\hat{F}(t, T)$ are given by the geometric Brownian motion in (44). This is, for example, when the risk-neutral dynamics of power prices $\hat{\Psi}_{t}$ in the absence of spikes are given by a geometric mean-reverting process in (12) so that $\hat{F}(t, T)$ is given in (43). Then, even in the presence of spikes in the power prices, when the time to maturity $T-t$ of the forward contract on power is relatively large with respect to the expected lifetime of a spike $\bar{t}_{s}=1 / a$, the risk-neutral dynamics of power forward prices $F(t, T)$ in (52) are also given by the geometric

Brownian motion in (44). This can be used to estimate the volatility of the geometric Brownian motion for the power forward prices $\hat{F}(t, T)$, as well as the volatility and the mean-reversion rate of the geometric mean-reverting process for the power prices $\hat{\Psi}_{t}$. This can also be used in the valuation and dynamic hedging of European contingent claims on forwards on power for power with spikes that will be discussed later in this paper.

Furthermore, consider a practically important special case of short-lived spikes when the expected lifetime of a spike $\bar{t}_{s}=1 / a$ is relatively short with respect to the expected time between spikes $\bar{t}_{r}=1 / b$.

Then, with the help of relationship (36), the ergodic average magnitude of spikes $\bar{\lambda}_{\text {erg }}$ given by (51) can be represented ${ }^{2,3}$ as follows

$$
\bar{\lambda}_{\text {erg }}=1+t_{c h}(\lambda-1)+o\left(t_{c h}\right)
$$

where $o\left(t_{c h}\right)$ stands for a term of order higher than $t_{c h}$.

With the help of relationships (52) and (53), the power forward prices $F(t, T)$ for power with spikes can be represented ${ }^{2,3}$ as the following correction to the corresponding power forward prices $\hat{F}(t, T)$ for power without spikes

$$
\begin{aligned}
F(t, T)\left(\hat{\Psi}_{t}\right)= & \hat{F}(t, T)\left(\hat{\Psi}_{t}\right) \\
& +t_{c h}(\lambda-1) \hat{F}(t, T)\left(\hat{\Psi}_{t}\right) \\
& +o\left(t_{c h}\right)
\end{aligned}
$$

Finally, the presented analysis in the special case of spikes with constant magnitude $\lambda$ and the time-homogeneous Markov process $M_{t}$ can be extended to the general case., ${ }^{2,3}$ In the case of the time-independent conditional probability density function $\Xi(\lambda)$, relationships (50)-(54) are still valid if the magnitude of spikes $\lambda$ is replaced by the conditional average magnitude of spikes $\bar{\lambda}$ given in (48).

\section{Valuation and hedging of European contingent claims on forwards on power for power with spikes}

Consider a practically important special case when the risk-neutral dynamics of power prices $\hat{\Psi}_{t}$ in the absence of spikes are given by a geometric mean-reverting process in (12). It will be shown that the value of a European contingent claim on forwards on power for power with spikes can be expressed in terms of the value of a European contingent claim on forwards on power in the Black-Scholes market environment. This allows for the dynamic hedging of European contingent claims on forwards on power for power with spikes 
via standard delta hedging.

For simplicity, a special case, when the spikes have constant magnitude $\lambda$, and the Markov process $M_{t}$ is time-homogeneous, is considered. It can be shown ${ }^{2}$ that owing to relationships (19), (43) and (52), the value $E(t, T, g)$ of a European contingent clam on power with spikes in (39) can be expressed in terms of the power forward price $F(t, T)$ for power with spikes

$$
\begin{aligned}
E(t, T, g)(F)= & \hat{E}_{\hat{\sigma}(t, T), 0}^{B S}\left(t, T, \bar{g}_{\text {erg }}\right)\left(F / \bar{\lambda}_{\text {erg }}\right) \\
& +O\left(\mathrm{e}^{-(T-t) a}\right)
\end{aligned}
$$

Moreover, it can be shown ${ }^{2}$ that the value $E(t, T, g)$ of a European contingent claim on forwards on power for power with spikes in (55) can be expressed in terms of the value of a European contingent claim on forwards on power in the Black-Scholes market environment

$$
\begin{aligned}
E(t, T, g)(F)= & \hat{E}_{\hat{\sigma}(t, T), 0}^{B S}\left(t, T, g_{\text {erg }}\right)(F) \\
& +O\left(\mathrm{e}^{-(T-t) a}\right)
\end{aligned}
$$

where $g_{\text {erg }}\left(\hat{\Psi}_{T}\right)=\bar{g}_{\text {erg }}\left(\hat{\Psi}_{T} / \bar{\lambda}_{\text {erg }}\right)$, and where the homogeneity of the values of European contingent claims in the Black-Scholes market environment was used.

Here, as discussed earlier in the paper, if the time to maturity $T-t$ of the forward contract on power is sufficiently large with respect to the expected lifetime of a spike $\bar{t}_{s}=1 / a$, the risk-neutral dynamics of power forward prices $F(t, T)$ for power with spikes are given by the geometric Brownian motion in (44). Therefore, relationship (56) allows for the dynamic hedging of European contingent claims on forwards on power for power with spikes via standard delta hedging.
For example, if the payoff $g$ is of the form (40), it can be shown ${ }^{2}$ with the help of the eigenclaims method that relationship (56) takes the following

form

$$
\begin{aligned}
& E(t, T, g)(F)=\sum_{n=1}^{N}\left[\left(\pi_{s} \lambda^{\omega_{n}}+\pi_{r}\right)\left(\pi_{s} \lambda+\pi_{r}\right)^{-\omega_{n}}\right. \\
& \left.\quad \times g_{n} \mathrm{e}^{(T-t)\left[1 / 2 \hat{\sigma}^{2}(t, T) \omega_{n}^{2}-\frac{1}{2}(t, T) \omega_{n}-\bar{r}(t, T)\right]} F^{\omega_{n}} \hat{\boldsymbol{\sigma}}^{2}\right] \\
& \quad+O\left(\mathrm{e}^{-(T-t) a}\right)
\end{aligned}
$$

If the payoff of a general European contingent claim on forwards on power for power with spikes can be suitably approximated, by payoffs $g$ in (40), its value in (56) can also be suitably approximated by $E(t, T, g)$ in the preceding relationship. Since $E(t, T, g)$ in the preceding relationship is determined analytically, the value of this general European contingent claim can also be approximated analytically. It is also pointed out ${ }^{2}$ that, in the case when the time-independent conditional probability density function $\Xi(\lambda)$ corresponds to a scaling probability distribution (that is, $\Xi(\lambda)$ is of the form $\gamma \lambda^{-\gamma-1}$ for some $\gamma>0$ ), then the proceeding relationship can be modified as follows

$$
\begin{aligned}
& E(t, T, g)(F)=\sum_{n=1}^{N}\left[\left(\pi_{s} \frac{\gamma}{\gamma-\omega_{n}}+\pi_{r}\right)\right. \\
& \quad \times\left(\pi_{s} \frac{\gamma}{\gamma-1}+\pi_{r}\right)^{-\omega_{n}} \\
& \left.\quad \times g_{n} \mathrm{e}^{(T-t)\left[1 / 2 \hat{\sigma}^{2}(t, T) \omega_{n}^{2}-1 / 2 \hat{\sigma}^{2}(t, T) \omega_{n}-\bar{r}(t, T)\right]} F^{\omega_{n}}\right] \\
& \quad+O\left(\mathrm{e}^{-(T-t) a}\right)
\end{aligned}
$$

where $\gamma>\max \left\{\omega_{n}, 1\right\}$.

Moreover, it can be shown ${ }^{2}$ that, in the case of the European call and put options with strike $X$, relationship (56) takes the 
following form

$$
\begin{aligned}
& C(t, T, F, X)= \\
& \quad \pi_{s}\left(\lambda / \bar{\lambda}_{\text {erg }}\right) \hat{C}_{\hat{\sigma}(t, T), 0}^{B S}\left(t, T, F,\left(\lambda / \bar{\lambda}_{\text {erg }}\right)^{-1} X\right) \\
& \quad+\pi_{r}\left(1 / \bar{\lambda}_{\text {erg }}\right) \hat{C}_{\hat{\sigma}(t, T), 0}^{B S}\left(t, T, F, \bar{\lambda}_{\text {erg }} X\right) \\
& \quad+O\left(\mathrm{e}^{-(T-t) a}\right) \\
& P(t, T, F, X)= \\
& \quad \pi_{s}\left(\lambda / \bar{\lambda}_{\text {erg }}\right) \hat{P}_{\hat{\sigma}(t, T), 0}^{B S}\left(t, T, F,\left(\lambda / \bar{\lambda}_{\text {erg }}\right)^{-1} X\right) \\
& \quad+\pi_{r}\left(1 / \bar{\lambda}_{\text {erg }}\right) \hat{P}_{\hat{\sigma}(t, T), 0}^{B S}\left(t, T, F, \bar{\lambda}_{\text {erg }} X\right) \\
& \quad+O\left(\mathrm{e}^{-(T-t) a}\right)
\end{aligned}
$$

Now consider a practically important special case of short-lived spikes when the expected lifetime of a spike $\bar{t}_{s}=1 / a$ is relatively short with respect to the expected time between spikes $\bar{t}_{r}=1 / b$. Then, with the help of relationships (37) and (53), the value in (55) - and hence in (56) - of the European contingent claim on forwards on power for power with spikes can be represented ${ }^{2}$ as the following correction to the value of the corresponding European contingent claim on forwards on power in the Black-Scholes market environment

$$
\begin{aligned}
& E(t, T, g)(F)= \\
& \quad \hat{E}_{\hat{\sigma}(t, T), 0}^{B S}\left(t, T, g_{\lambda_{T}=1}\right)(F) \\
& \quad+t_{c h}\left(\hat{E}_{\hat{\sigma}(t, T), 0}^{B S}\left(t, T, g_{\lambda_{T}=\lambda}-g_{\lambda_{T}=1}\right)(F)\right. \\
& \left.\quad-(\lambda-1) F \Delta_{\hat{\sigma}(t, T), 0}^{B S}\left(t, T, g_{\lambda_{T}=1}\right)(F)\right) \\
& \quad+o\left(t_{c h}\right)
\end{aligned}
$$

where $\Delta_{\hat{\sigma}(t, T), 0}^{B S}\left(t, T, g_{\lambda_{T}=1}\right)$ is the delta of the European contingent claim with the value $\hat{E}_{\hat{\sigma}(t, T), 0}^{B S}\left(t, T, g_{\lambda_{T}=1}\right)$, that is

$$
\begin{aligned}
& \Delta_{\hat{\sigma}(t, T), 0}^{B S}\left(t, T, g_{\lambda_{T}=1}\right)(F) \\
& \quad=\frac{\partial}{\partial F} \hat{E}_{\tilde{\sigma}(t, T), 0}^{B S}\left(t, T, g_{\lambda_{T}=1}\right)(F)
\end{aligned}
$$

For example, in the case of the European call and put options with strike $X$, relationship (57) takes the following form $^{2}$

$$
\begin{aligned}
C(t, T, F, X)= & \hat{C}_{\hat{\sigma}(t, T), 0}^{B S}(t, T, F, X) \\
& +t_{c h}\left[\lambda \left(\hat{C}_{\hat{\sigma}(t, T), 0}^{B S}\left(t, T, F, \lambda^{-1} X\right)\right.\right. \\
& -\hat{C}_{\hat{\sigma}(t, T), 0}^{B S}(t, T, F, X) \\
& \left.-(\lambda-1) F \Delta_{c, \hat{\sigma}(t, T), 0}^{B S}(t, T, F, X)\right] \\
& +o\left(t_{c h}\right)
\end{aligned}
$$

and

$$
\begin{aligned}
P(t, T, F, X)= & \hat{P}_{\hat{\sigma}(t, T), 0}^{B S}(t, T, F, X) \\
& +t_{c h}\left[\lambda \hat{P}_{\hat{\sigma}(t, T), 0}^{B S}\left(t, T, F, \lambda^{-1} X\right)\right. \\
& -\hat{P}_{\hat{\sigma}(t, T), 0}^{B S}(t, T, F, X) \\
& \left.-(\lambda-1) F \Delta_{p, \hat{\sigma}(t, T), 0}^{B S}(t, T, F, X)\right] \\
& +o\left(t_{c h}\right)
\end{aligned}
$$

where the deltas $\Delta_{c, \tilde{\sigma}(t, T), 0}^{B S}(t, T, F, X)$ and $\Delta_{p, \hat{\sigma}(t, T), 0}^{B S}(t, T, F, X)$ in the Black-Scholes market environment of the European call and put options with strike $X$ are given by

$$
\begin{aligned}
& \Delta_{c, \tilde{\sigma}(t, T), 0}^{B S}(t, T, F, X)=\mathrm{e}^{-\bar{r}(T, t)(T-t)} N\left(d_{+}\right) \\
& \Delta_{p, \hat{\sigma}(t, T), 0}^{B S}(t, T, F, X)=\mathrm{e}^{-\bar{r}(T, t)(T-t)}\left(N\left(d_{+}\right)-1\right)
\end{aligned}
$$

with $N\left(d_{+}\right)$given in (21). (See, for example, Wilmott $\left.{ }^{14}\right)$.

Finally, the analysis of the special case of spikes with constant magnitude $\lambda$ and the time-homogeneous Markov process $M_{t}$ presented here can be extended to the general case. ${ }^{2}$ In the case of the time-independent conditional probability density function $\Xi(\lambda)$, relationships (56) - (57) are still valid if - in view of relationships (32) and (51) $-g_{\lambda_{T}=\lambda}$ is replaced by the conditional average $\bar{g}$ of the payoff $g_{\lambda_{t}}$ over the magnitude of spikes $\lambda_{T}$ at time $T$ given in (27) and the magnitude 
of spikes $\lambda$ is replaced by the conditional average magnitude of spikes $\bar{\lambda}$ given in (48).

\section{EXTENSIONS OF THE MODEL}

The model for power prices with spikes and for the valuation of European contingent claims on power presented in this paper can be extended in the following ways.

- Both positive and negative multiplicative spikes, as well as additive spikes and spikes of more complex shapes, can be considered. ${ }^{1,2}$

- The assumption that the spike process and the process for power prices in the absence of spikes are independent can be relaxed. ${ }^{1,2}$ Moreover, the spike process can be replaced with an arbitrary suitable Markov process, or even a finite product of arbitrary suitable Markov processes. This includes, as a special case, a multi-state Markov process and the product of several two-state Markov processes. For example, in view of the interpretation of the non-Markovian process for power prices with spikes in terms of the structural model discussed above, a multi-state Markov process can be used to model spikes in power prices as a result of partial outages and intermittent operation of a representative power plant. In turn, a product of several two-state Markov processes can be used to model spikes in power prices as a result of failure of multiple power plants.

- European contingent claims on power with spikes and other commodities, whose prices do not exhibit spikes, can also be valued. Those include fuel-sensitive, weather-sensitive and load-sensitive contingent claims such as spark spread options and full requirements contracts. ${ }^{31,32}$

- European contingent claims on power at several distinct points on the grid with spikes in all power prices can also be valued. These include transmission options. ${ }^{33,34}$

- Contingent claims of a general type, such as universal contingent claims on power with spikes, can also be valued with the help of the semilinear evolution equation for universal contingent claims. ${ }^{2,18}$ These include Bermudan and American options. Universal contingent claims and the semilinear evolution equation for universal contingent claims were introduced by the author in previous works. ${ }^{11,23-28}$

\section{Acknowledgments}

The author thanks Manu Asthana, Ehud Ronn, Vince Kaminski, Alexander Eydeland, Helyette Geman, Mark Garman, Les Clewlow, Chris Strickland, Ilia Bouchouev, Peter Nance, Frank Li, Fred Guan, Ivo Steijn, Wang Chiu and Milan Lukic for their attention to this work. I also thank Lisa Bloomer for her help in editing this article. Saving the best for last, I thank my wife Larisa and my son Nikita for their love, patience and care.

\section{References}

1 Kholodnyi, V. A. (2000) 'The Stochastic Process for Power Prices with Spikes and Valuation of European Contingent Claims on Power', Preprint, TXU Energy Trading.

2 Kholodnyi, V. A. (2000) 'A Non-Markovian 
Process for Power Prices with Spikes and Valuation of Contingent Claims on Power', Preprint, TXU Energy Trading.

3 Kholodnyi, V. A. (2000) 'Modeling Power Forward Prices for Power with Spikes', Preprint, TXU Energy Trading.

4 Kholodnyi, V. A. (2001) 'A non-Markov method', Energy and Power Risk Management Magazine, March, pp. 20-24.

5 Kholodnyi, V. A. (2004) 'Valuation and hedging of European contingent claims on power with spikes: A non-Markovian approach', Journal of Engineering Mathematics, Vol. 49, No. 3, pp. 233-252.

6 Ethier, R. and Dorris, G. (1999) 'Do not ignore the spikes', Energy and Power Risk Management Magazine, July/August, pp. 31-33.

7 Clewlow, L., Strickland, C. and Kaminski, V. (2000) 'Jumping the gaps', Energy and Power Risk Management Magazine, December, pp. 26-27.

8 Johnson, B. and Barz, G. (1999) 'Selecting Stochastic Processes for Modeling Electricity Prices', in 'Energy Modeling and the Management of Uncertainty', Risk Publications, London, pp. 3-21.

9 Jailet, P., Ronn, E. and Tompaidis, S. (1998) 'The quest for valuation', Energy and Power Risk Management Magazine, June, pp. 14-16.

10 Dollard, J. D. and Friedman, C. N. (1979) 'Product Integration', Addison-Wesley, Reading, MA.

11 Kholodnyi, V. A. (1995) 'On the Linearity of Bermudan and American Options with General Time-Dependent Payoffs in Partial Semimodules', Preprint, Integrated Energy Services.

12 Kholodnyi, V. A. (2000) 'Valuation of a Swing Option in the Mean-Reverting Market Environment', Preprint, TXU Energy Trading.

13 Kholodnyi, V. A. (2001) 'Analytical valuation in a mean-reverting world', Energy and Power Risk Management Magazine, August, pp. 40-45.

14 Wilmott, P. (1998) 'Derivatives', Wiley, New York, NY.

15 Kholodnyi, V. A. (2002) 'Analytical Valuation of a Full Requirements Contract as a Real Option by the Method of Eigenclaims', in Ronn, E. I. (Ed.), 'Real Options and Energy Management', Risk Publications, London, UK, pp. 635-658.

16 Kholodnyi, V. A. (1995) 'Beliefs-Preferences Gauge Symmetry Group and Replication of Contingent Claims in a General Market Environment', Preprint, Integrated Energy Services.

17 Kholodnyi, V. A. (1998) 'Beliefs-Preferences Gauge Symmetry Group and Replication of
Contingent Claims in a General Market

Environment', IES Press, Research Triangle Park, NC.

18 Kholodnyi, V. A. (2000) 'Valuation and Dynamic Replication of Contingent Claims on Power with Spikes in the Framework of the Beliefs-Preferences Gauge Symmetry', Preprint, TXU Energy Trading.

19 Kholodnyi, V. A. (2002) 'Valuation and dynamic replication of contingent claims in the framework of the beliefs-preferences gauge symmetry', European Physical Journal B, Vol. 27, No. 2, pp. 229-238.

20 Kholodnyi, V. A. (2002) 'Valuation and dynamic replication of contingent claims in a general market environment based on the beliefs-preferences gauge symmetry', Russian Journal of Mathematical Physics, Vol. 9, No. 2, pp. 161-179.

21 Kholodnyi, V. A. (2003) 'Beliefs-preferences gauge symmetry and dynamic replication of contingent in a general market environment', Journal of the Dynamics of Continuous, Discrete and Impulsive Systems B, Vol. 10, No. 1, pp. 81-94.

22 Kholodnyi, V. A. 'Introduction to the Beliefs-Preferences Gauge Symmetry', Elsevier Science, Amsterdam, the Netherlands, forthcoming.

23 Kholodnyi, V. A. (1995) 'Universal Contingent Claims', Preprint, Integrated Energy Services.

24 Kholodnyi, V. A. (1995) 'Semilinear Evolution Equation for Universal Contingent Claims', Preprint, Integrated Energy Services.

25 Kholodnyi, V. A. (1997) 'A nonlinear partial differential equation for american options in the entire domain of the state variable', Journal of Nonlinear Analysis, Vol. 30, No. 8, pp. 5059-5070.

26 Kholodnyi, V. A. (1997) 'A Semilinear Evolution Equation for General Derivative Contracts', in Price, J. F. (Ed.), 'Derivatives and Financial Mathematics', Nova Science Publishers, Commack, NY, pp. 119-138.

27 Kholodnyi, V. A. (2003) 'Universal Contingent Claims and Multiplicative Measures: Examples and Applications', in Sivasundaram, S. (Ed.), 'Proceedings of the 4th International Conference on Nonlinear Problems in Aviation and Aerospace', European Conference Publications, Cambridge, UK, pp. 259-270.

28 Kholodnyi, V. A. (2005) 'Universal Contingent Claims in a General Market Environment and Multiplicative Measures', in Sambandham, M. 
(Ed.), 'Proceedings of the 4th International

Conference on Dynamical Systems and

Applications', Dynamic Publishers, Atlanta, GA, pp. 206-212.

29 Dynkin, E. (1965) 'Markov Processes', Springer, Berlin, Germany.

30 Cohen-Tannoudji, C., Diu, B. and Laloe, F. (1997) 'Quantum Mechanics', Wiley, New York, NY.

31 Kholodnyi, V. A. (2000) 'Valuation of a Swing Option on Power with Spikes', Preprint, TXU
Energy Trading.

32 Kholodnyi, V. A. (2000) 'Valuation of a Spark Spread Option on Power with Spikes', Preprint, TXU Energy Trading.

33 Kholodnyi, V. A. (2000) 'Valuation of European Contingent Claims on Power at Two Distinct Points on the Grid with Spikes in Both Power Prices', Preprint, TXU Energy Trading.

34 Kholodnyi, V. A. (2000) 'Valuation of a Transmission Option on Power with Spikes', Preprint, TXU Energy Trading. 